\title{
P2X7 Receptors and TMEM16 Channels Form a Hub with Implications for Macropore Formation and Current Facilitation
}

\section{Kate Dunning}

University of Strasbourg https://orcid.org/0000-0001-9811-7087

Adeline Martz

University of Strasbourg

Francisco Peralta

University of Strasbourg https://orcid.org/0000-0002-0727-1706

\section{Federico Cevoli}

University of Strasbourg

\section{Eric Boué-Grabot}

Université de Bordeaux

Antoine Taly

UMR 7199 CNRS - Université

Vincent Compan

University of Montpellier

Fanny Gautherat

University of Strasbourg

Patrick Wolf

University of Strasbourg

Thierry Chataigneau

UMR 7199 CNRS, Unistra

Thomas Grutter ( $\nabla$ grutter@unistra.fr )

University of Strasbourg

\section{Article}

Keywords: P2X7 ion channels, anoctamin, ATP sensitization, cell permeabilization.

Posted Date: February 4th, 2021

DOl: https://doi.org/10.21203/rs.3.rs-153271/v1 
License: (c) (i) This work is licensed under a Creative Commons Attribution 4.0 International License. Read Full License 
4 Kate Dunning ${ }^{1 \dagger}$, Adeline Martz ${ }^{1 \dagger}$, Francisco Andrés Peralta ${ }^{1,2}$, Federico Cevoli ${ }^{1}$, Eric 5 Boué-Grabot ${ }^{3,4}$, Antoine Taly ${ }^{5,6}$, Vincent Compan $^{7,8}$, Fanny Gautherat ${ }^{1}$, Patrick Wolf ${ }^{1}$, 6 Thierry Chataigneau ${ }^{1}$, Thomas Grutter ${ }^{1,2 *}$

7 'University of Strasbourg, Centre National de la Recherche Scientifique, CAMB UMR 8 7199, F-67000 Strasbourg, France. ${ }^{2}$ University of Strasbourg Institute for Advanced 9 Studies (USIAS), Strasbourg, France. ${ }^{3}$ Université de Bordeaux, Institut des Maladies 10 Neurodégénératives, UMR 5293, F-33000 Bordeaux, France. ${ }^{4}$ CNRS, Institut des Maladies 11 Neurodégénératives, UMR 5293, F-33000 Bordeaux, France. ${ }^{5}$ CNRS, Université de Paris, 12 UPR 9080, Laboratoire de Biochimie Théorique, 13 rue Pierre et Marie Curie, F-75005, 13 Paris, France. ${ }^{6}$ Institut de Biologie Physico-Chimique-Fondation Edmond de Rothschild, PSL Research University, Paris, France. ${ }^{7}$ Institut de Génomique Fonctionnelle (IGF), University of Montpellier, CNRS, INSERM, Montpellier, France. ${ }^{8}$ Laboratoire d'Excellence Canaux Ioniques d'Intérêt Thérapeutique (LabEx ICST), Montpellier, France.

$17 \dagger$ These authors contributed equally to this work.

18 * Correspondence author: Thomas Grutter. Email: grutter@unistra.fr

19 ORCIDs: https://orcid.org/0000-0002-4351-9203

21 Classification: Biological Science

22 Keywords: P2X7 ion channels; anoctamin; ATP sensitization; cell permeabilization.

23 Author Contributions: K.D., and T.G. designed research; K.D., A.M., F.A.P., F.C., E.B24 G., A.T., V.C., F.G., and T.C. performed research; K.D., A.M., F.A.P., E.B-G., A.T., P.W., 25 T.C., and T.G. analyzed data; T.G. and K.D. wrote the paper with contributions from all 26 the authors.

27 This PDF file includes:

28 Main Text

29 Figures 1 to 8 


\section{$30 \quad$ Abstract}

31 P2X7 receptors (P2X7) are cationic channels involved in many diseases. They exhibit 32 unique behaviors, such as "macropore" formation, which corresponds to enhanced large 33 molecule cell membrane permeability, and current facilitation, which is caused by 34 prolonged activation. These two phenomena have often been confounded, but thus far no 35 clear mechanisms have been resolved. Here we provide evidence that current facilitation 36 and macropore formation involve functional complexes comprised of P2X7 and TMEM16,

37 a family of $\mathrm{Ca}^{2+}$-activated ion channel/scramblases. We found that current facilitation 38 results in an increase of complex-embedded P2X7 open channel probability, a result 39 mimicked by plasma membrane cholesterol depletion. We further show that macropore 40 formation entails two distinct large molecule permeation components, one of which 41 requires protein complexes featuring TMEM16F subtype, the other likely being direct 42 permeation through the P2X7 pore itself. Such protein complexes can be considered to 43 represent a regulatory hub intimately involved in distinct P2X7 functionalities. 
Main Text

\section{Introduction}

Purinergic $\mathrm{P} 2 \mathrm{X} 7$ receptors (P2X7) are members of the $\mathrm{P} 2 \mathrm{X}$ receptor family, a class of membrane proteins forming trimeric channels activated by extracellular ATP ${ }^{1}$. They are expressed in different cell types, mainly in immune and glial cells. Following ATP gating, P2X7 initiates nonselective metal cation flux $\left(\mathrm{Na}^{+}, \mathrm{K}^{+}\right.$and $\left.\mathrm{Ca}^{2+}\right)$, which in turn triggers distinct activation pathways, such as the secretion of pro-inflammatory cytokines or modulation of cell death. Owing to its key role in multiple pathologies, including chronic inflammation, neurodegeneration, neuropathic pain, metabolic diseases, rheumatoid arthritis, Crohn's disease and cancer, P2X7 has become a particularly relevant therapeutic target, sparking intense interest for drug development ${ }^{2}$.

Among the P2X family, P2X7 exhibits several hallmark features, including an elongated intracellular C-terminus, the necessity of unusually high concentrations of ATP required for channel activation, and ATP-evoked current facilitation (also known as ATP sensitization $)^{3}$. This process is caused by repeated or prolonged ATP applications, which yield a leftward shift of agonist affinity ${ }^{4,5}$, and is inhibited by cholesterol ${ }^{6,7}$, an essential component of the cell membrane. Recent work has further indicated that this inhibition may be counteracted by the presence of palmitoylated cysteine residues located in the intracellular C-terminus of the $\mathrm{P} 2 \mathrm{X} 7$, suggesting that lipid composition may represent a powerful means to allosterically regulate receptor function ${ }^{8}$.

In addition to ATP-evoked current facilitation, "macropore" formation or cell permeabilization is another hallmark of $\mathrm{P} 2 \mathrm{X} 7^{3,9}$. This process allows the passage of nanometer-sized molecules $(<900 \mathrm{Da})$ across the cell membrane, and is usually monitored by the cellular uptake of fluorescent dyes $^{10}$, such as ethidium and YO-PRO-1. First described in 1979 in mast cells ${ }^{11}$, and later confirmed in various other cell lines, the activation of this pathway eventually leads to cell death ${ }^{9}$. Often confounded with the ATPevoked current facilitation phenomenon ${ }^{12,13}$, several hypotheses have been proposed to explain macropore formation, including possible interactions with the pore forming pannexin hemichannel ${ }^{14}$, but none have proved entirely satisfactory. One hypothesis that has remained prevalent until very recently was the so-called "pore dilation" theory" which postulated that ATP stimulation induces a progressive, time-dependent expansion of the pore, gradually increasing both current and large molecule permeability. This idea was appealing in its simplicity, but has been challenged by recent work ${ }^{17}$, and a new permeation mechanism supported by biochemical ${ }^{8}$, electrophysiological ${ }^{17-20}$, photochemical ${ }^{20}$ and modeling ${ }^{17,20}$ data has emerged, in which the passage of small cationic molecules, such as $N$-methyl-D-glucamine $\left(\mathrm{NMDG}^{+}\right)$and spermidine, occurs immediately after ATP gating, via a non-dilated, open channel state. The main difference resides in the permeation rates, whereby $\mathrm{NMDG}^{+}$ions flow at a reduced rate compared to that of $\mathrm{Na}^{+20}$.

At first glance, this mechanism appears to reconcile most of the data ${ }^{21}$, but there remains an unanswered question as to how nanometer-sized molecules larger than $\mathrm{NMDG}^{+}$ 
(195 Da), such as YO-PRO-1 (376 Da) or even larger dyes (e.g. YOYO-1, $763 \mathrm{Da})^{22}$, can directly permeate the $\mathrm{P} 2 \mathrm{X} 7$ channel itself ${ }^{23,24}$. While reconstruction of an engineered $\mathrm{P} 2 \mathrm{X} 7$, truncated at the $\mathrm{N}$ - and $\mathrm{C}$-termini, into artificial liposomes supported the hypothesis that the pore itself is capable of YO-PRO-1 dye uptake, recent cryoelectron microscopy (cryoEM) structures of ATP-bound, full-length rat P2X7 revealed that the open channel diameter measures only $5 \AA$ (ref. ${ }^{25}$ ), an aperture that seems too narrow to allow the passage of nanometer-sized molecules. Given that the minimal cross-section of YO-PRO- 1 is $\sim 7 \AA$, it is therefore hard to conceive that cellular uptake of molecules similar to or larger than YOPRO-1 occurs solely through the P2X7 open pore itself, suggesting that additional routes are possible ${ }^{23}$. Given the importance of $\mathrm{P} 2 \mathrm{X} 7$ in numerous pathologies, there is an urgent need to understand the molecular foundations of macropore formation and current facilitation, which undoubtedly remain two of the most enigmatic P2X features.

Recently, $\mathrm{Ca}^{2+}$-activated $\mathrm{Cl}^{-}$channels $(\mathrm{CaCCs})$ have been suggested to constitute one such possible route. Pharmacological inhibition of these channels prevents dye uptake in different cell lines, including HEK293 cells ${ }^{26}$, human and mouse macrophages ${ }^{22,26}$ as well as human microglia ${ }^{27}$. In addition, a functional coupling between these channels and P2X7 has also been described in Xenopus oocytes ${ }^{28}$. In mouse macrophages, the transmembrane protein 16F (TMEM16F or Anoctamin-6) was identified as one of these $\mathrm{CaCCs}$, and proposed to mediate effects essential for innate immunity downstream of $\mathrm{P} 2 \mathrm{X} 7^{26}$. TMEM16F belongs to the TMEM16 family, a vast group of membrane proteins characterized by a high degree of functional diversity, including roles such as $\mathrm{Ca}^{2+}$ activated $\mathrm{Cl}^{-}$channels, $\mathrm{Ca}^{2+}$-dependent phospholipid scramblases, and dual function nonselective ion channel/phospholipid scramblases ${ }^{29}$. There are ten TMEM16 proteins (TMEM16A-K, excluding I) found in humans, and recent evidence suggests that TMEM16F functions as a dual $\mathrm{Ca}^{2+}$-activated non-selective (rather than purely $\mathrm{Cl}^{-}$) ion channel and $\mathrm{Ca}^{2+}$-dependent phospholipid scramblase. This moonlight scramblase activity led to the suggestion that TMEM16F activation by ATP-evoked P2X7 $\mathrm{Ca}^{2+}$ flux mediates downstream intracellular signaling processes, resulting in outcomes such as membrane blebbing and apoptosis ${ }^{26}$. However, despite a possible direct interaction of P2X7 and TMEM16F having been evoked ${ }^{26}$, no molecular complex has been identified thus far, and any significance relating to macropore formation and current facilitation remains to be elucidated.

In this work, we set out to test the hypothesis that P2X7 and TMEM16 form functional complexes in HEK293T cells and Xenopus oocytes. We combined mutagenesis techniques, genetic approaches, biochemical experiments, single-channel and whole-cell recordings, confocal microscopy, live cell imaging and molecular modeling to show that these complexes have implications in both agonist-evoked current facilitation and macropore formation. Our data thus offer new insights into these enigmatic processes that have proved difficult to explain for 40 years. 


\section{Results}

\section{ATP-evoked current facilitation results in increased P2X7 open channel probability}

To investigate the mechanism of current facilitation at the molecular level, we recorded by patch-clamp electrophysiology single-channel activity from outside-out patches of HEK293T cells transiently transfected with rat P2X7 (rP2X7). To avoid nonspecific channel activation evoked by millimolar concentrations of $\mathrm{ATP}^{30}$, we used the more potent P2X7 agonist 2'(3')-O-(4-benzoylbenzoyl)ATP (BzATP), which activates the channel in the low $\mu \mathrm{M}$ range, and reduced the amount of extracellular divalent cation concentrations to dampen P2X7 channel inhibition ${ }^{31}$. In control patches, barely visible BzATP-gated single-channel openings and closings were detected when data were filtered at a corner frequency $\left(f_{c}\right)$ of $1 \mathrm{kHz}($ Fig. S1A). However, by further filtering data at 100 $\mathrm{Hz}$, clearly resolved unitary currents were observed that rapidly disappeared upon BzATP washout (Fig. 1A, left, and Fig. S1A and B). Under such conditions, the dead time $\left(\mathrm{t}_{\mathrm{d}}\right)$ was equal to $1.8 \mathrm{~ms}$, and consequently transitions shorter than $t_{d}$ were ignored. These unitary currents originated from $\mathrm{P} 2 \mathrm{X} 7$ because no currents were recorded in the absence of BzATP, in the presence of the highly selective P2X7 antagonist AZ10606120 co-applied with BzATP, nor in non-transfected cells stimulated with BzATP (Fig. 1A, right, and Fig. S1C and D).

Next, we devised a protocol in which current facilitation was first induced by a 30 s perfusion of BzATP before establishing an excised membrane patch in the outside-out configuration (Fig. 1B). We first verified that such long agonist perfusion induced a strong whole-cell current facilitation that is similar to eight repeated short agonist applications (Fig. S1F and G), as previously described ${ }^{6}$. We observed some variability in the degree of whole-cell current facilitation and a significant increase in the deactivation time constant of facilitated currents during BzATP washout (Fig. S1H and I), two features that were previously reported ${ }^{6,32}$.

For control condition (no BzATP perfusion), two unitary current amplitudes were observed (Fig. 1C, top), in agreement with previous data obtained with ATP from the human (hP2X7) expressed in oocytes ${ }^{33}$. As we selected only those patches containing three or fewer channels for analysis (see Materials and Methods), we confirm that these currents did not originate from stackings of double openings, but rather reflect a dual gating mechanism ${ }^{33}$. The simultaneous presence of these two discrete conductance levels $(\mathrm{O} 1=$ $1.89 \pm 0.26 \mathrm{pS} ; \mathrm{O} 2=3.39 \pm 0.44 \mathrm{pS}, \mathrm{n}=7$ patches, Fig. $1 \mathrm{D}$, top) was observed in 5 out of 7 patches (two patches exhibited only the $\mathrm{O} 2$ state). Analysis of open and shut-time histograms fitted with multiple exponentials revealed a mean open time for O1 state $\left(\tau_{\mathrm{O} 1}\right)$ of $24 \mathrm{~ms}$ and mean open time for $\mathrm{O} 2$ state $\left(\tau_{\mathrm{O} 2}\right)$ of $44 \mathrm{~ms}$, and a mean closed time $\left(\tau_{\mathrm{C}}\right)$ of $185 \mathrm{~ms}$ (Fig. 1E, top and Fig. S1E, left, 1,518 events).

In patches having undergone a $30 \mathrm{~s}$ BzATP facilitation treatment, the two unitary current amplitudes were still observed (in 5 out of 6 patches), and conductance values were similar to those of untreated conditions $(P=0.279$ for $\mathrm{O} 1$ and 0.383 for $\mathrm{O} 2$, unpaired Student's $t$-test, Fig. $1 \mathrm{C}$ and $\mathrm{D}$, middle, $\mathrm{n}=6$ patches). However, a dramatic increase of the 
mean open times of both conductance levels was recorded $\left(\sim 10\right.$-fold for $\mathrm{O} 1, \tau_{\mathrm{O} 1}=234 \mathrm{~ms}$, and $\sim 5$-fold for $\mathrm{O} 2, \tau_{\mathrm{O} 2}=211 \mathrm{~ms}$ ), along with a modest increase of the mean closed time $\left(\sim 1.5\right.$-fold, $\tau_{\mathrm{C}}=279 \mathrm{~ms}$, Fig. 1E, middle and Fig. S1E, right, 754 events $)$. As a result, the open channel probability ( $\mathrm{N} P \mathrm{O}$ ) for both conductance states combined increased by $\sim 2.9$ fold, from 0.17 to 0.47 . Of note is that this increase is not only in the same range as BzATPevoked current facilitation observed in the whole-cell configuration (between $\sim 3$ - to $\sim 4$ fold), but also consistent with the increased deactivation time constant after facilitation. This suggests that in HEK293T cells, agonist-evoked current facilitation predominantly originates from a large increase of rP2X7 open channel probability, but not of singlechannel conductance, thereby disfavoring the pore dilation theory.

\section{Plasma membrane cholesterol depletion increases P2X7 open channel probability}

To uncover the molecular basis of current facilitation, we recorded BzATP-gated unitary currents in excised outside-out patches in which membrane cholesterol was acutely depleted by methyl- $\beta$-cyclodextrin (MCD), a cholesterol chelating agent (Fig. 1B). It has been shown that MCD potentiates P2X7 function, by dramatically enhancing whole-cell currents evoked by the first agonist application ${ }^{6}$. As a result, initial currents recorded from MCD-treated cells are of significantly greater magnitude than those recorded in control cells, showing instead similar magnitude to control cell currents recorded after BzATP facilitation. After verifying that this was effectively the case in the whole-cell configuration (Fig. S1J and K), we recorded single-channel currents from outside-out patches excised from cells that were pre-treated with MCD. Similarly to BzATP-evoked current facilitation, we found that MCD treatment enhanced mean open times of both conductance states, when compared to control patches ( $\sim 16$-fold for $\mathrm{O} 1, \tau_{\mathrm{O} 1}=378 \mathrm{~ms}$ and $\sim 3$-fold for $\mathrm{O} 2, \tau_{\mathrm{O} 2}=134 \mathrm{~ms}, 396$ events $)$, with a small but statistically insignificant reduction of unitary current amplitudes $(P=0.146$ for $\mathrm{O} 1$ and 0.080 for O2, unpaired Student's $t$-test, Fig. 1C-E, bottom; all patches contained both $\mathrm{O} 1$ and $\mathrm{O} 2$ states, except two patches that exhibit only the $\mathrm{O} 2$ state, $\mathrm{n}=7$ patches). In line with deactivation kinetics of facilitated current, MCD treatment also significantly increased the deactivation time constant of BzATP-evoked currents, an effect observed from the first application (Fig. S1H and I). These data demonstrate that MCD recapitulates ATP-evoked current facilitation, presumably by dissociating cholesterol from the receptor.

Taken together, these results show that prolonged activation of P2X7 enhances open channel probability, an effect mimicked by depletion of plasma membrane cholesterol.

\section{A functional interplay between P2X7 and TMEM16}

Having shown that current facilitation results in an increase of the single-channel open probability, we next asked whether this increase is related to macropore formation. 
Because $\mathrm{CaCC}$ inhibitors have been previously shown to inhibit P2X7-dependent dye uptake $^{22,26}$, we tested whether they are able to inhibit P2X7 currents which have been facilitated by four repeated applications of BzATP. We focused on tannic acid (TA), flufenamic acid (FFA) and 9-anthracene-carboxylic acid (9-AC), for which functional modulation of TMEM16 activity, including TMEM16A ${ }^{34,35}$, TMEM16B ${ }^{36}$ and TMEM16 ${ }^{26}$, has been recently shown in recombinant systems. We found that application of $20 \mu \mathrm{M}$ TA alone for $60 \mathrm{~s}$ (referred to as "application alone") to facilitated receptors abolished subsequent BzATP-evoked whole-cell currents in rP2X7-expressing HEK293T cells, while $1 \mathrm{mM} 9$-AC reduced subsequent currents by $\sim 35 \%$ (Fig. 2A and B). For FFA $(100 \mu \mathrm{M})$, no inhibition was found in these conditions, however, a single co-application of FFA and BzATP at the end of FFA perfusion reduced subsequent BzATP-evoked currents to $\sim 35 \%$ (Fig. 2A and B). Contrary to TA and 9-AC, FFA inhibitions were fully reversible upon washout. Importantly, no inward current was induced by application of TA, 9-AC, or FFA alone (Fig. S2A). Thus, these data suggest that facilitated P2X7 currents are fully inhibited by TA, and partially inhibited by 9 -AC and FFA.

To exclude a possible direct modulation of P2X7 activity by selected CaCC inhibitors, we investigated the effects of these inhibitors in Axolotl oocytes (Ambystoma mexicanum), which are reported to be electrophysiologically void of $\mathrm{CaCCs}^{37,38}$. Using two-electrode voltage-clamp (TEVC) and the "application alone" protocol, no P2X7 current inhibition was detected by 9 -AC or FFA (Fig. 3A and B), while 50\% inhibition was observed for both inhibitors in Xenopus oocytes (Fig. 3C and D), which do express endogenous $\mathrm{CaCCs}^{37}$. Note that for FFA, inhibition took place using the "application alone" protocol directly, suggesting that washout dissociates bound FFA more slowly in Xenopus oocytes than in HEK293T cells. Current facilitation was observed in Xenopus oocytes, albeit not systematically, but not in Axolotl oocytes, suggesting that the absence of current facilitation is not correlated to an absence of $\mathrm{CaCC}$ inhibition. These data, therefore, strongly suggest that 9-AC and FFA do not act as inhibitors of rP2X7 itself, suggesting that their partial inhibition of facilitated rP2X7 currents in HEK293T cells and Xenopus oocytes occurs through a functional interplay between endogenous human $(\mathrm{h})$ hTMEM16 and rP2X7 that we named thereafter P2X7/TMEM16.

However, in contrast to 9-AC and FFA, TA abolished currents in Axolotl oocytes (Fig. 3A and B), as well as in Xenopus oocytes (Fig. 3C and D), suggesting that it may directly inhibit the $\mathrm{P} 2 \mathrm{X} 7$ channel itself through specific or non-specific mechanisms. The action of TA is therefore unclear, but we have decided to include the results throughout this manuscript to further document its action on P2X7.

\section{P2X7/TMEM16 contributes to current facilitation}

Next, we asked whether CaCC inhibitors applied after the very first BzATP application in naïve cells prevent current facilitation. Inhibition was monitored by comparing currents evoked by repeated BzATP applications in the presence or absence of CaCC inhibitors. We found that TA and 9-AC not only strongly inhibited BzATP-evoked 
currents (measured at the fourth application), but also prevented current facilitation (Fig. 2C and D). FFA was not different from the control, and accordingly no inhibition was detected (Fig. 2D). Given the fact that 9-AC has been shown to not act on P2X7 itself, the above data strongly suggest that the functional interplay between P2X7 and TMEM16 contributes to current facilitation.

\section{The functional interaction of P2X7/TMEM16 is retained in excised patches}

Next, we asked whether inhibitions observed in the whole-cell configuration are retained in excised patches. Co-applications of BzATP and each of the $\mathrm{CaCC}$ inhibitors to rP2X7-expressing outside-out patches inhibited BzATP-evoked single-channel currents to levels that were similar to those obtained in the whole-cell configuration when inhibitors were applied before facilitation (Fig. 2E and F). The only exception was FFA, for which no or very weak inhibition was observed in the whole-cell configuration while inhibition was detected in excised patches (Fig. 2F and Fig. S2B). The reason is unclear, but may be related to differences between the whole-cell and single-channel modes of recording. Single-channel inhibitions were reversible upon washout, even for TA, and levels of inhibition were similar to those obtained by co-applying the selective P2X7 inhibitor AZ10606120 with BzATP. Overall, 9-AC inhibition data indicate that the interplay between $\mathrm{rP} 2 \mathrm{X} 7$ and hTMEM16 remains stable in excised patches, and therefore does not require an intact cell and associated cellular components.

Noticeably, 9-AC and FFA inhibitors largely decreased the frequency at which we observed BzATP-evoked single-channel openings, but not the unitary conductance of those few currents which remained (Fig. 2E), thereby suggesting that these compounds do not act as fast P2X7 open channel blockers.

\section{P2X7/TMEM16 contributes partially to YO-PRO-1 uptake}

Having shown the existence of functional interaction between rP2X7 and hTMEM16, we next asked whether this interaction is involved in dye uptake in HEK293T cells. We used YO-PRO-1, a fluorescent dye of $376 \mathrm{Da}$, and showed robust dye uptake in living cells expressing rP2X7 following $10 \mu \mathrm{M}$ BzATP application (Fig. 4A and B). No dye uptake was observed in the absence of BzATP or in its presence in non-transfected cells (Fig. 4C). TA co-applied with BzATP abolished dye uptake, but curiously, its extent of inhibition was greater than that of controls (i.e. without BzATP or in non-transfected cells) (Fig. 4A-C). This suggests that TA, in addition to its dye uptake inhibition, may quench YO-PRO-1 fluorescence before dye uptake. To test this hypothesis, we acquired emission spectra of YO-PRO-1 bound to double strand DNA (dsDNA) in the absence or presence of TA, and found that addition of TA led to immediate and complete quenching of fluorescence intensity (Fig. 4D). In contrast, no fluorescence quenching was observed with 9-AC or FFA (Fig. 4D), even 15 minutes after inhibitor addition. These data 
demonstrate that TA is a powerful YO-PRO-1 quencher, and therefore should not be used as a pharmacological inhibitor in dye uptake assays.

Next, we found that 9-AC significantly reduced BzATP-stimulated YO-PRO-1 uptake, by $\sim 40 \%$ while no dye uptake inhibition was observed with FFA (Fig. 4A-C). These results suggest that the functional interaction between P2X7 and TMEM16 contributes partially to dye uptake. Because TMEM16F was previously shown to mediate functional effects downstream of P2X7 (ref. ${ }^{26}$ ), we test the hypothesis that the P2X7/TMEM16 involved in dye uptake in HEK293T cells may feature hTMEM16F subtype.

\section{TMEM16F underlies a part of YO-PRO-1 uptake}

To investigate the participation of hTMEM16F in dye uptake, we applied CRISPR/Cas9 gene editing to produce a hTMEM16F-deficient (16F-null) HEK293T cell line (Fig. S3). We verified the absence of endogenously expressed hTMEM16F in this cell line compared to wild-type (WT) HEK293T cells by Western blot (Fig. 5A). Before addressing dye uptake, we first tested whether rP2X7 expression is influenced by endogenous hTMEM16F, by comparing rP2X7 subunit expression in both HEK293T and $16 \mathrm{~F}$-null cell lines. We used a $\mathrm{rP} 2 \mathrm{X} 7$ construct that was tagged at its $\mathrm{C}$-terminus with a myc sequence, and found a significant increase of both total and cell surface expression of rP2X7 subunit in 16F-null cell lines compared to HEK293T cells (Fig. 5B and C). These data reveal that the presence of endogenous hTMEM16F channels reduces $\mathrm{rP} 2 \mathrm{X} 7$ subunit expression when this latter is ectopically expressed in HEK293T cells.

Next, we measured BzATP-induced dye uptake in the rP2X7-expressing 16F-null cell line. A significant $\sim 40 \%$ reduction of YO-PRO-1 uptake was detected compared to HEK293T cells, while controls in the absence of BzATP showed no dye uptake (Fig. 5D$\mathrm{G})$. Of note, the extent of reduction of dye uptake observed in 16F-null cells was similar to that observed pharmacologically with 9-AC in HEK293T cells (compare Fig. 5E and Fig. $4 \mathrm{~B})$. These genetic data thus unambiguously demonstrate a statistically significant contribution of hTMEM16F to dye uptake.

Nevertheless, dye uptake was not abolished in 16F-null cells, raising the possibility that the remaining dye uptake involves other TMEM16 members and/or intrinsic passage through the P2X7 pore. To further probe these possibilities, we asked whether $\mathrm{CaCC}$ inhibitors were able to inhibit this remaining dye uptake. Co-application of FFA did not inhibit BzATP-induced YO-PRO-1 uptake in rP2X7R-expressing 16F-null cells (Fig. 5G), as previously observed in HEK293T cells. Interestingly, 9-AC no longer inhibited dye uptake either (Fig. 5G), suggesting that the inhibition of cell permeabilization observed with 9-AC in HEK293T cells was related to hTMEM16F (Fig. 4C). These results suggest that $9-\mathrm{AC}$ is a selective inhibitor of hTMEM16F channels involved in dye uptake, and support the hypothesis that remaining dye uptake is likely related to the direct passage through the P2X7 pore. 
To further validate TMEM16F contribution to dye uptake, we asked whether reexpression of TMEM16F along with P2X7 in 16F-null cell lines is able to rescue dye uptake. By assaying varying cDNA ratios between $\mathrm{rP} 2 \mathrm{X} 7$ and rat TMEM16F (rTMEM16F), we found that a ratio of 1:0.05 rP2X7 to rTMEM16F ( $2 \mu \mathrm{g}$ rP2X7 and 0.1 $\mu \mathrm{g}$ rTMEM16F) fully rescued dye uptake (Fig. 5F). These results therefore not only confirm the contribution of TMEM16F to dye uptake, regardless of the species (human or rat), but also reveal an optimal non-stoichiometric cDNA ratio for restoring dye uptake. Although the underlying mechanism is elusive, these data are coherent with our findings showing reduced $\mathrm{rP} 2 \mathrm{X} 7$ expression in the presence of TMEM16F, which may explain why a cDNA ratio largely skewed in favor of $\mathrm{P} 2 \mathrm{X} 7$ is needed to rescue dye uptake.

\section{P2X7 and TMEM16F form protein complexes}

To confirm a close physical proximity of P2X7 and TMEM16F, we carried out coimmunoprecipitation in 16F-null cells overexpressing myc tagged rP2X7 and rTMEM16F that was also tagged at its $\mathrm{C}$-terminus with a myc-DDK sequence. Because we found that dye uptake was fully rescued at a cDNA ratio of 1:0.05 in 16F-null cells, and given the fact that P2X7 expression is modulated by the presence of TMEM16F, we asked whether varying this ratio from 1:0.05 to 1:1 may also influence protein expression. We found that co-expressing $\mathrm{rP} 2 \mathrm{X} 7$ and $\mathrm{rTMEM} 16 \mathrm{~F}$ at a $1: 1$ ratio dramatically reduced total $\mathrm{rP} 2 \mathrm{X} 7$ expression in 16F-null cells, but not at a 1:0.05 ratio compared to control (Fig. 6A). Thus, in agreement with our previous dye uptake data, these results further confirm the negative effect of TMEM16F on $\mathrm{rP} 2 \mathrm{X} 7$ expression, and suggest that the ratio 1:0.05 is more suitable for co-immunoprecipitation. In these conditions, we found that anti-FLAG antibody, which recognizes DDK tag of myc-DDK tagged rTMEM16F, specifically and efficiently coimmunoprecipitated rP2X7 subunits, despite rTMEM16F expression being 20 times less than that of $\mathrm{rP} 2 \mathrm{X} 7$ (Fig. 6B). These data therefore indicate a close physical proximity between rTMEM16F and $\mathrm{rP} 2 \mathrm{X} 7$.

Next, we asked whether the two proteins co-localize in $16 \mathrm{~F}$-null cells by confocal microscopy. We designed a fluorescent $\mathrm{rP} 2 \mathrm{X} 7$ that was tagged at its C-terminus with mScarlet, a bright monomeric red fluorescent protein, and transiently co-expressed rP2X7mScarlet with rTMEM16F at a ratio of 1:0.05 in 16F-nulls cells. By subsequently staining rTMEM16F with a primary anti-h16F antibody coupled to a secondary Alexa 488 conjugated antibody, we showed that both proteins fully co-localized in $16 \mathrm{~F}$-null cells (Pearson's coefficient $=0.897 \pm 0.014 ; \mathrm{n}=8$ cells from 5 transfections; Fig. 6C). Although most of the co-localization appears to be intracellular, putative plasma membrane colocalization was also detected (Fig. 6C).

Taken together, these data are consistent with the idea that P2X7 and TMEM16F form protein complexes.

\section{TMEM16F shapes P2X7 unitary conductance}


Having revealed the existence of $\mathrm{P} 2 \mathrm{X} 7 / \mathrm{TMEM} 16 \mathrm{~F}$ protein complexes, we next asked whether P2X7 functions, other than those involved in dye uptake, are also affected in $16 \mathrm{~F}$-null cells. We found that current facilitation remained similar to that recorded in HEK293T cells, albeit exhibiting seemingly slower kinetics, as well as current density (Fig. 7A, left and middle). A similar variability in the degree of current facilitation was also noticed (Fig. 7A, right), as well as a significant increase in the deactivation time constants of facilitated currents (Fig. S4A and B). Equally, no change in the profile of whole-cell inhibition by $\mathrm{CaCC}$ inhibitors was observed compared to that of HEK293T cells (Fig. 7B and $\mathrm{C}$ and Fig. S4C and D). These data indicate that the absence of hTMEM16F has no apparent impact on whole-cell current properties.

However, single-channel recordings revealed the disappearance of the $\mathrm{O} 2$ conductance state observed in HEK293T cells, while the O1 conductance state remained present in 7 out of 9 patches (Fig. 7D and E). In the remaining 2 patches, an additional unitary current of greater magnitude than that corresponding to the $\mathrm{O} 2$ conductance state was recorded. However, as this current occurred only very occasionally, we cannot attribute it definitively (Fig. 7D and E). As a result of these changes in unitary currents, the mean single-channel conductance was significantly lower in $16 \mathrm{~F}$-null cells than in HEK293T cells (Fig. 7F). This decrease in mean single-channel conductance may compensate for the increased cell surface expression of P2X7 subunit in 16F-null cell lines (Fig. 5C), thus accounting for the apparently unchanged whole-cell current density. In addition, these unitary currents were strongly inhibited by TA and AZ10606120, but less so by FFA and 9-AC (Fig. 7G). Collectively, our data suggest that endogenous hTMEM16F shapes the $\mathrm{O} 2$ single-channel conductance recorded in $\mathrm{rP} 2 \mathrm{X} 7$-expressing HEK293T cells.

\section{Molecular modeling supports experimental data}

Finally, we produced molecular models of the P2X7/TMEM16F complex by using recent cryo-EM structures of the full-length $\mathrm{rP} 2 \mathrm{X} 7$ solved in the absence or presence of ATP in detergent ${ }^{25}$, and of the full-length mouse TMEM16F (mTMEM16F) solved as a homodimer in a $\mathrm{Ca}^{2+}$-bound state in lipid nanodiscs ${ }^{39}$. We generated models in which one rP2X7 trimer was docked to one mTMEM16F dimer, with no assumption on the stoichiometry and relative position. After positioning the proteins in the membrane plane, they were allowed to move within this plane and along the z-axis in a Monte-Carlo procedure. Dockings were carried out without a priori, except for their initial position in the membrane, and models were ranked based on energy-scoring. This procedure produced two complex models, one comprised of the P2X7 open channel state and the other comprised of the P2X7 closed channel state (Fig. 8A and Fig. S5). These modeling data are therefore in agreement with experimental data indicating that P2X7 and TMEM16F are able to form a physical association within the membrane. 


\section{Discussion}

In this work, we shed new light on the molecular mechanisms underlying macropore formation and agonist-evoked current facilitation, two hallmarks of P2X7 that have for several decades remained enigmatic. We reveal that both phenomena implicate functional complexes formed between P2X7 and members of the TMEM16 family that we named P2X7/TMEM16. We also provide evidence that, contrary to current beliefs ${ }^{12,13}$, current facilitation does not stem from pore dilation.

The first important finding of this study is that when ectopically expressed in HEK293T cells, P2X7 forms protein complexes with TMEM16F channels (and possibly with other members of the TMEM16 family). We also reveal a functional coupling between P2X7 and CaCCs in Xenopus oocytes, but no conclusive evidence can be provided on the existence of such complexes in oocytes. A previous work has provided evidence that such protein complexes are not formed in Xenopus oocytes, although a similar functional coupling has been described between P2X7 and TMEM16A, but not with TMEM16F ${ }^{28}$. The reason of this discrepancy is unclear, but may be related to a very low surface expression of TMEM16F and/or to looser protein complexes in oocytes. However, in HEK293T cells it appears that these protein complexes formed with TMEM16F have important implications for $\mathrm{P} 2 \mathrm{X} 7$ expression and function. We show that TMEM16F influences $\mathrm{P} 2 \mathrm{X} 7$ expression, possibly by retaining $\mathrm{P} 2 \mathrm{X} 7$ within intracellular compartments, suggesting that TMEM16F may act as a regulating element of $\mathrm{P} 2 \mathrm{X} 7$ cell surface expression. Furthermore, we show that native TMEM16F contributes to the function of $\mathrm{P} 2 \mathrm{X} 7$, through the occurrence of an $\mathrm{O} 2$ conducting state. The significance of this state is unknown, but currents originating from the $\mathrm{O} 2$ state likely do not stem from TMEM16F channel, since the activity of this outwardly-rectifying channel is expected to be minimal at negative potentials ${ }^{39,40}$. One possibility could be that the close physical proximity of TMEM16F to $\mathrm{P} 2 \mathrm{X} 7$ influences single-channel conductance characteristics (Fig. 8A), but the underlying molecular mechanism is currently unknown.

The existence of such protein complexes also provides a rational explanation of how P2X7 current inhibition can be mediated by the non-selective 9-AC inhibitor. The lack of action of 9-AC on BzATP-induced P2X7 currents in CaCC-void Axolotl oocytes provides strong evidence that this inhibitor does not directly bind to P2X7, but instead binds to endogenous TMEM16 channels and inhibits P2X7 activity via an allosteric interaction due to the close proximity of the two channels. Our data further reveal its selective inhibition of TMEM16F channels involved in dye uptake (Fig. 8B). Yet, the fact that 9-AC no longer inhibits dye uptake in 16F-null cells, while still inhibiting BzATPevoked currents, suggests that another TMEM16 subtype is coupled to P2X7 ion channel function, including current facilitation, but not to dye uptake. Importantly, because 9-AC does not abolish BzATP-evoked currents, even after a 1-min long application (Fig. 2C), the possibility that a fraction of "free" P2X7 channels (i.e. those not embedded in a complex) resides at the cell membrane cannot be excluded (Fig. 8B). 
Conversely, the mode of action of FFA and TA remains unclear. Although FFA inhibition was observed in Xenopus oocytes with no direct action on P2X7, its inhibitory effect was quite variable in HEK293T cells, and no definitive conclusion can be drawn. For TA, however, P2X7 currents were systematically abolished following application of the inhibitor, even in Axolotl oocytes, suggesting that it may either directly inhibit P2X7 channel or act non-specifically through a currently unknown mechanism. Regardless of the mechanism at play, this result is in apparent disagreement with previous work having found that co-application of TA and ATP to human microglia did not inhibit endogenous P2X7 channel activity ${ }^{27}$. The reasons behind this discrepancy are unknown, but may result from factors differing between recombinant and native systems. Finally, our data demonstrate that TA is not suitable for dye uptake experiments because of its fluorescence quenching properties, a finding that is also supported by a very recent study ${ }^{41}$.

The second important finding of the present work is that P2X7/TMEM16 largely contributes to current facilitation. This is supported by the fact that 9-AC strongly impairs BzATP-evoked current facilitation. Interestingly, TMEM16 channels were also previously suggested to contribute to current facilitation in Xenopus oocytes through a different mechanism ${ }^{28}$. The authors suggested that current facilitation results in the secondary activation of TMEM16A Cl- channels that follows P2X7 activation and subsequent $\mathrm{Ca}^{2+}$ flux. However, we show that in HEK293T cells the mechanism underlying current facilitation results in a dramatic increase of $\mathrm{P} 2 \mathrm{X} 7 \mathrm{NPO}$, with no evidence of the appearance of a new, secondary unitary conductance. Therefore, it appears that different mechanisms underlying current facilitation are at play depending on the cell type. The two mechanisms can co-exist, but at present we do not know whether those TMEM16 $\mathrm{Cl}^{-}$channels are active within complexes.

In close agreement with previous data ${ }^{7,8}$, our results suggest that plasma membrane cholesterol is a negative allosteric modulator of P2X7. It maintains the complex-embedded P2X7, and possibly "free" P2X7, in a low channel activity state in response to initial, brief agonist application. Longer or repeated agonist application then switches channels to higher activity states, which, in turn, underlies the observed increased current amplitudes and slowed deactivation kinetics. As suggested previously ${ }^{6,8}$, we too propose that this effect is likely attributable to a progressive dissociation of cholesterol from channels (Fig. 8B). Molecular mechanisms underlying agonist-evoked cholesterol dissociation remain currently elusive, but one possibility would be an activity-dependent increase of membrane mobility of complexes from high (e.g. lipid raft) to low cholesterol content ${ }^{42}$. Whether cholesterol binds directly to P2X7, TMEM16 channels or the complex as a whole, remains to be determined. A recent study has demonstrated that cholesterol directly inhibits $\mathrm{P} 2 \mathrm{X} 7$, most likely through interactions with membrane palmitoyl moieties attached to cysteine residues located in an intracellular juxtamembrane cysteine-rich domain of P2X7 (ref. ${ }^{8}$ ). Supported by $\mathrm{P} 2 \mathrm{X} 7$ cryo-EM structures ${ }^{25}$, one possibility is that cholesterol binding to those competing transmembrane sites interferes with P2X7 channel gating, most likely by stabilizing the closed channel state, and/or by destabilizing the open channel state. 
The third important finding of this study is that large molecule permeability proceeds through at least two components in HEK293T cells (Fig. 8B). The first one likely entails a direct passage through the $\mathrm{P} 2 \mathrm{X} 7$ pore, as suggested previously ${ }^{8,24}$. Cryo-EM structures of P2X7 suggest the existence of a finite $\sim 5 \AA$ open channel diameter, with no evidence of larger sized pores ${ }^{25}$. The minimal cross-section of YO-PRO- 1 being $\sim 7 \AA$, which exceeds the $5 \AA$-outer limit of the P2X7 open pore, suggests that direct passage of YO-PRO-1 would occur at extremely low rates, as recently suggested in the case of P2X2 (ref. ${ }^{20}$ ). We therefore suggest that molecules smaller than YO-PRO-1, such as $\mathrm{NMDG}^{+}$, robustly diffuse through the $\mathrm{P} 2 \mathrm{X} 7$ open pore, as revealed previously ${ }^{20}$, albeit at slower permeation rates than those of $\mathrm{Na}^{+}$. However, for larger molecules, such as YOYO-1 (763 Da) or TO-TO-1 (894 Da) for which P2X7-dependent cell permeabilization mechanisms have been described ${ }^{22,43}$, direct permeation through the resolved P2X7 open channel structure seems challenging ${ }^{25}$. Therefore, the contribution of a second, non-exclusive component that involves TMEM16F channels (and perhaps other TMEM16 members) represents an alternative permeation mechanism of larger nanometer-sized molecules (Fig. $8 \mathrm{~B})$. Importantly, as this second component is linked to a weakly expressed TMEM16F relative to P2X7, our data may explain why knockdown of TMEM16F by siRNAs, for which low expression levels can escape silencing, does not apparently affect P2X7 dye uptake in macrophages ${ }^{22}$ and HEK cells ${ }^{26}$. Our data thus provide a possible explanation for previous data that have remained otherwise difficult to explain ${ }^{9,11,22,23}$. In light of our results showing that long agonist exposure increases P2X7 NPo, we suggest that components 1 and 2 are boosted during long agonist exposure, which both contribute to the enhanced levels of large molecule cell membrane permeability observed in dye uptake experiments (Fig. 8B).

The precise permeation pathway underlying large molecule permeation of component 2 is currently unknown, but may involve the TMEM16F pore as it functions as a poorly selective ion channel ${ }^{40,44}$, enabling the passage of small organic cations, such as $\mathrm{NMDG}^{+}$(ref. ${ }^{40}$ ). Another possibility is that dye uptake shares the same mechanisms as those implicated in TMEM16F scramblase activity ${ }^{39,40,45}$. Whatever the mechanism, identification of the dye uptake pathway, as well as the molecular arrangement and stoichiometry of P2X7/TMEM16F merits further study.

In conclusion, our data reveal that P2X7 can form functional complexes with TMEM16 channels in HEK293T cells. Interestingly, a very recent work has also reported similar modulation of P2X7 activity with another channel, TMEM163 (ref. ${ }^{46}$ ). We propose that P2X7/TMEM16 forms a regulating hub, which, upon P2X7 activation and dependent on surrounding membrane cholesterol level, orchestrate a hive of activity, eliciting not only channel gating (e.g. efflux of $\mathrm{K}^{+}$and $\mathrm{Ca}^{2+}$ signaling), but also other cell-specific signaling, including membrane blebbing, interleukin release and phospholipid scrambling ${ }^{9}$. Given the critical roles of $\mathrm{P} 2 \mathrm{X} 7$ and TMEM16 channels in many diseases, this platform may potentially be involved in important pathophysiological processes that lead, for example, to inflammation ${ }^{47}$ and mechanical allodynia ${ }^{48,49}$, and may therefore be therapeutically important. Future work is needed to unravel its broader implications in disease. 
542

543

544

545

\section{Materials and Methods}

\section{Cell culture and transfection}

HEK293T cells (ATCC) were cultured in Dulbecco's modified Eagle's medium, supplemented with $10 \%$ fetal bovine serum, 1x GlutaMax, 100 units $/ \mathrm{mL}$ penicillin and 100 $\mu \mathrm{g} / \mathrm{mL}$ streptomycin (Gibco Life Technologies). For HEK293T hTMEM16F-null cells, this medium was further supplemented with $1 \mu \mathrm{g} / \mathrm{mL}$ puromycin dihydrochloride (Gibco Life Technologies).

Trypsin-treated cells were seeded onto $9-12 \mathrm{~mm}$ glass coverslips (VWR) in 35mm dishes for patch-clamp and fluorescence experiments, or in $100 \mathrm{~mm}$ dishes for biochemical experiments, in both cases pre-treated with poly-L-lysine (Sigma Aldrich). Cells were incubated at $37^{\circ} \mathrm{C}$ and in presence of $5 \% \mathrm{CO}_{2}$.

Transfections were carried out using the calcium phosphate precipitation method. The cDNA encoding rat P2rx7 gene (ID Q64663) constructs and enhanced Green Fluorescent Protein construct (eGFP) were contained within pcDNA3.1(+) plasmids (Invitrogen). $\mathrm{rP} 2 \mathrm{X} 7$-mScarlet was designed by tagging $\mathrm{mScarlet}$ (from gene synthesis) in the C-terminus of $\mathrm{rP} 2 \mathrm{X} 7$ within a pcDNA3.1(+) vector. rTMEM16F cDNA was contained within a pCMV6 vector featuring a myc-DDK tag in the C-terminus (OriGene, RR212483). This myc-DDK tag containing the sequence DYKDDDDK can be recognized by anti-FLAG antibody. For whole-cell patch clamp experiments, cells were co-transfected with the rP2X7 construct $(0.5-0.8 \mu \mathrm{g})$ and an eGFP $(0.3 \mu \mathrm{g})$ which allowed to identify cells having undergone efficient transfection. For single-channel experiments, the quantity of transfected $\mathrm{rP} 2 \mathrm{X} 7$ construct DNA was reduced to $0.01-0.08 \mu \mathrm{g}$. Cells were washed one day after transfection with PBS solution, and the medium replaced with fresh. For biochemical experiments, each $100 \mathrm{~mm}$ dish was either co-transfected with cDNAs encoding myc tagged $\mathrm{rP} 2 \mathrm{X} 7(5 \mu \mathrm{g})$ and myc-DDK tagged rTMEM16F constructs $(5$ or $0.25 \mu \mathrm{g}$, as indicated) or transfected with each construct alone $(10 \mu \mathrm{g})$. For dye uptake rescue experiments, cells were transfected with $2 \mu \mathrm{g}$ myc tagged $\mathrm{rP} 2 \mathrm{X} 7$ construct either alone or co-transfected with $1,0.1,0.01$ or $0.002 \mu \mathrm{g}$ myc tagged rTMEM16F. For colocalization by confocal microscopy, cells were transfected with $2 \mu \mathrm{g}$ myc tagged $\mathrm{rP} 2 \mathrm{X} 7$ and $0.1 \mu \mathrm{g}$ myc tagged rTMEM16F.

\section{Oocytes preparation and injection}

Ovarian lobes were surgically removed from Xenopus laevis and Ambystoma mexicanum Axolotl females and oocytes were isolated as described ${ }^{50,51}$. After nuclear injection of 300 pg of rat P2X7 construct, oocytes were incubated in Barth's solution containing $1.8 \mathrm{mM}$ $\mathrm{CaCl}_{2}$ and gentamycin $(10 \mathrm{mg} / \mathrm{ml})$ at $19^{\circ} \mathrm{C}$ for $1-3$ days before electrophysiological recordings.

\section{Ethics approval}

All experimental procedures complied with official European guidelines for the care and use of laboratory animals (Directive 2010/63/UE) and were approved by the ethical committee of Bordeaux. All animals were treated humanely and with regard for alleviation of suffering. 


\section{Molecular biology}

CRISPR/Cas9 method was carried out as previously described ${ }^{52,53}$. The oligonucleotide encoding the sgRNA sequence (5'-AATAGTACTCACAAACTCCG-3'), which targets exon 2 of TMEM16F gene, containing BbsI overhangs was cloned into BbsI sites in pSpCas9(BB)-2A-Puro (Addgene plasmid ID:48139). The plasmid obtained was transfected into HEK293T using the calcium phosphate precipitation method. After $48 \mathrm{~h}$ of transfection, $1 \mu \mathrm{g} / \mathrm{mL}$ puromycin was applied to select cells for $72 \mathrm{~h}-96 \mathrm{~h}$, with medium change every $24 \mathrm{~h}$. Then, transfected cells were serial-diluted in 96-well plates to select for single-cell colonies. After 14-21 days, the single-cell colonies were expanded and screened for the absence of hTMEM16F. Verification was carried out by sequencing (Eurofins Genomics, see Fig. S3) and Western blot with the rabbit anti-hTMEM16F antibody (Sigma Aldrich, HPA038958) (see Fig. 5A).

\section{Patch-clamp electrophysiology}

Whole-cell recordings were carried out $24 \mathrm{~h}-48 \mathrm{~h}$ after transfection, and single-channel recordings $24 \mathrm{~h}$ after transfection. Patch pipettes were pulled from borosilicate glass capillaries (Harvard Apparatus) to yield resistances of 3-5 M $\Omega$ for whole-cell recordings, and 15-20 M $\Omega$ for single-channel recordings. For single-channel recordings, pipettes were coated with Sylgard 184 (Dow Corning Co.) and fire polished before use. $\mathrm{pH}$ was verified for all solutions used, and if necessary, adjusted carefully using $\mathrm{NaOH}$ to $\mathrm{pH}$ 7.32-7.33. All solutions were maintained at approximately $300 \mathrm{mOsm}$.

Cells were voltage-clamped to $-60 \mathrm{mV}$ (whole-cell) or $-120 \mathrm{mV}$ (outside-out) using the EPC10 amplifier (HEKA), and data were recorded with PATCHMASTER software. Data were acquired at $10 \mathrm{kHz}$ and low-pass filtered at $2.9 \mathrm{kHz}$. Applications of agonist and/or inhibitor were carried out by perfusion, using three capillary tubes placed directly over the cell/patch of interest. These capillaries are displaced using the SF-77B Perfusion Fast Step system (Warner), ensuring solution exchange within 5-10 ms. For whole-cell recordings, Normal Extracellular Solution (NES) contained $140 \mathrm{mM} \mathrm{NaCl}, 2.8 \mathrm{mM} \mathrm{KCl}, 1 \mathrm{mM} \mathrm{CaCl}$, $0.1 \mathrm{mM} \mathrm{MgCl}_{2}, 10 \mathrm{mM}$ HEPES, $10 \mathrm{mM}$ Glucose, $\mathrm{pH}$ 7.32-7.33. Patch pipettes contained $140 \mathrm{mM} \mathrm{KCl}, 5 \mathrm{mM} \mathrm{MgCl}$, $5 \mathrm{mM}$ EGTA, $10 \mathrm{mM}$ HEPES, adjusted to $\mathrm{pH} 7.3$ with $\mathrm{NaOH}$. For single-channel recordings, NES contained $147 \mathrm{mM} \mathrm{NaCl}, 2 \mathrm{mM} \mathrm{KCl}, 1 \mathrm{mM} \mathrm{CaCl}$, $0.1 \mathrm{mM} \mathrm{MgCl} 2,10 \mathrm{mM}$ HEPES, $10 \mathrm{mM}$ Glucose adjusted to $\mathrm{pH} 7.3$ with $\mathrm{NaOH}$. Intracellular solution contained $147 \mathrm{mM} \mathrm{NaF}, 10 \mathrm{mM}$ HEPES and $10 \mathrm{mM}$ EGTA, adjusted to $\mathrm{pH} 7.3$.

2'(3')-O-(4-benzoylbenzoyl) adenosine 5'-triphosphate (BzATP) (triethylammonium salt, Sigma Aldrich) was used as P2X7 agonist at a concentration of $10 \mu \mathrm{M}$. TMEM16 inhibitors used were the following: tannic acid (TA) $20 \mu \mathrm{M}$, flufenamic acid (FFA) $100 \mu \mathrm{M}$, 9anthracene-carboxylic acid (9-AC) $1 \mathrm{mM}$. Specific P2X7 antagonist AZ10606120 (dihydrochloride salt, Tocris) was used at a concentration of $1 \mu \mathrm{M}$. BzATP and TA were solubilized in NES. In the case of AZ10606120, FFA and 9-AC, concentrated stock solutions were firstly produced in DMSO which were subsequently diluted in NES to achieve the desired working concentration in $\leq 0.1 \%$ DMSO. For control application (without inhibitors), $0.1 \%$ DMSO was added in NES. Owing to the facilitation effect 
produced by prolonged agonist exposure, only one cell was patched per coverslip, to ensure that receptors studied are indeed "naïve" upon the first agonist stimulation. For cells and patches treated by methyl- $\beta$-cyclodextrin (MCD), coverslips were incubated with $50 \mu \mathrm{L}$ of $15 \mathrm{mM}$ MCD (Sigma Aldrich) solubilized in DMEM for 15 minutes at $37^{\circ} \mathrm{C}$. This volume ensures complete coverage of the coverslip with MCD solution. Following incubation, MCD solution is removed by gentle washing with NES, before being used for electrophysiological experiments. In facilitated conditions, perfusion of BzATP $(10 \mu \mathrm{M})$ was carried out for $30 \mathrm{~s}$ while in the cell-attached configuration, before piercing of the membrane and excising of the patch to the outside-out configuration, in which recordings were carried out.

\section{Xenopus and Axolotl oocyte electrophysiology}

TEVC recordings were performed as previously described ${ }^{50,51}$. Briefly, recordings were carried out at room temperature using glass pipettes (1-2 M $\Omega$ ) filled with $3 \mathrm{M} \mathrm{KCl}$ solution to ensure reliable holding potentials. Oocytes were voltage-clamped at $-60 \mathrm{mV}$ and membrane currents were recorded with an OC-725B amplifier (Warner Instruments) and digitized at $1 \mathrm{kHz}$ on a Power PC Macintosh G4 using Axograph X. Oocytes were perfused at a flow rate of $10-12 \mathrm{ml} / \mathrm{min}$ with Ringer solution, $\mathrm{pH} 7.4$ containing in $\mathrm{mM}: 115 \mathrm{NaCl}$, $3 \mathrm{NaOH}, 2 \mathrm{KCl}, 1 \mathrm{CaCl}_{2}$, and $10 \mathrm{HEPES}$. BzATP and TMEM16 inhibitors were solubilized in Ringer solution and used at the same concentration as in patch-clamp experiments. Agonist and drugs were applied using a computer-driven valve system (Ala Scientific).

\section{Co-immunoprecipitation, cell surface biotinylation assays and Western blotting}

For co-immunoprecipitation, non-transfected or transfected 16F-null cells with myc tagged rP2X7 and myc-DDK tagged rTMEM16F at a ratio of 1:0.05, were lysed by incubation with gentle agitation for 80 minutes at $4^{\circ} \mathrm{C}$ with lysis buffer containing $20 \mathrm{mM}$ HEPES, $100 \mathrm{mM} \mathrm{NaCl}, 1 \%$ Triton-X, $5 \mathrm{mM}$ EDTA, Pierce Protease Inhibitor Tablets (Thermo Fischer). After 10 minutes of centrifugation at 14,000 rpm the supernatant was collected. Input samples were collected at this stage. After an initial pre-clearing process of cell lysate with Protein G Sepharose Fast Flow resin (Sigma Aldrich, see manufacturer's instructions), $5 \mu \mathrm{g}$ anti-FLAG mouse antibody (Sigma Aldrich, F1804) was added to the cell lysate and left under gentle agitation for 2 hours at $4^{\circ} \mathrm{C}$. Protein $\mathrm{G}$ Sepharose Fast Flow resin was then added and left under gentle agitation for $1 \mathrm{~h}$ at $4{ }^{\circ} \mathrm{C}$. Following this, the resin was washed three times with washing buffer containing $20 \mathrm{mM}$ HEPES, $500 \mathrm{mM} \mathrm{NaCl}, 1 \%$ Triton-X, $5 \mathrm{mM}$ EDTA, Pierce Protease Inhibitor Tablets (Thermo Fischer), and two times with lysis buffer. The resin was then resuspended in NuPage LDS Sample Buffer x1 (Thermo Fischer) and $70 \mathrm{mM}$ DTT, and boiled for 10 minutes. The supernatant was loaded onto NuPage Novex Bis-Tris 4-12\% gel (Thermo Fischer) and migrated in MOPS buffer. Transfer onto nitrocellulose membrane was carried out using the TransBlot Turbo system (BioRad) and the membrane blocked for 30 minutes in TPBS (PBS supplemented by $1 \%$ dried non-fat mik, $0.5 \%$ BSA, $0.05 \%$ Tween-20). The membrane was incubated overnight at $4{ }^{\circ} \mathrm{C}$ with TPBS containing either anti-c-myc mouse antibody, dilution 1:500 (Invitrogen, Thermo Fischer, reference 13-2500) or anti- $\beta$-actin mouse antibody, dilution 1:5,000 (Sigma Aldrich, reference A5441). Three washes with TPBS were carried out, before a second incubation in TPBS containing HRP-conjugated sheep anti-mouse antibody, 
dilution 1:10,000 (GE Life Sciences, reference NA9310) for 1-2 hours at room temperature. Three further washes with TPBS were carried out before revelation using Amersham ECL Select Western Blotting Detection Reagent (GE Life Sciences). Chemiluminescence was measured using the Amersham Imager 600. Images have been cropped for presentation; original uncut images are available in Fig. S6.

For cell surface biotinylation, transfected HEK293T and 16F-null cells were washed three times with PBS+ (PBS supplemented with $1 \mathrm{mM} \mathrm{MgCl}$ and $0.4 \mathrm{mM} \mathrm{CaCl}$, adjusted to $\mathrm{pH}$ 8.0), and incubated 30 minutes with $2 \mathrm{mM}$ sNHS-SS-Biotin (Thermo Fisher, reference 21331) in PBS+. Then, a washout step with PBS+ was carried out, before quenching any excess sNHS-SS-Biotin for 10 minutes with $20 \mathrm{mM}$ Tris in PBS+. Three further washing steps with PBS were carried out before lysis step. Following lysis (as described above), neutravidin-agarose resin (Thermo Fisher, reference 29200) was added to the cell lysate and incubated under gentle agitation overnight at $4^{\circ} \mathrm{C}$. Samples were then prepared as described for co-immunoprecipitation and loaded onto Mini-PROTEAN TGX 4-15\% and migrated in TGS buffer. Western blotting was carried out as described above.

\section{Immunocytochemistry}

Transfected or non-transfected $16 \mathrm{~F}$-null cells were fixed $24 \mathrm{~h}$ post transfection in $4 \%$ PFA (PBS, 4\% paraformaldehyde, $\mathrm{pH} 8.0$ ) for $15 \mathrm{~min}$ at room temperature. Coverslips were then washed in PBS for 5 min and permeabilized for $30 \mathrm{~min}$ in block solution (PBS, 1\% BSA, $0.1 \%$ Triton X-100). Coverslips were incubated at $4^{\circ} \mathrm{C}$ overnight with primary antihTMEM16F rabbit antibody (Sigma Aldrich, HPA038958) diluted 1:500 in block solution. Coverslips were then washed in PBS and incubated with secondary fluorescent probeconjugated antibody (goat anti-rabbit IgG H\&L (Alexa Flour® 488) (ab150077, Abcam) diluted 1:500, and Hoescht stain $(4 \mu \mathrm{g} / \mu \mathrm{L})$ for $1 \mathrm{~h}$ at RT in block solution. Coverslips were then mounted using mounting reagent (Prolong Gold antifade, P36930, Invitrogen) and allowed to dry overnight prior to imaging with Leica SPE.

\section{Fluorescence microscopy}

Video fluorescence microscopy measurements were carried out using a Leica FW4000 and 40x objective (Platform of Quantitative Imagery, Faculté de Pharmacie, Université de Strasbourg). Acquisition of fluorescence images was carried out at an interval of every 5 seconds using the software MetaMorph (Molecular Devices). The experiment was divided into two acquisition periods; an initial acquisition of 10 minutes where cells were incubated in NES/YO-PRO-1 solution where YO-PRO-1 is at a concentration of $10 \mu \mathrm{M}$ (iodide salt, Thermo Fischer). The solution was then gently exchanged for a NES/YO-PRO-1 solution containing BzATP and inhibitors (or BzATP alone) for a second acquisition period of 15 minutes. This second solution containing inhibitors was prepared immediately prior to application, and concentrations used are the same as those used for electrophysiology. Cells were maintained at $37^{\circ} \mathrm{C}$ during measurements.

Confocal imaging was captured with Leica SPE using oil immersion objective: $63 x$, Numerical Aperture 1.4. Excitation $(\lambda$ exc) and emission $(\lambda \mathrm{em})$ wavelengths were as follows: Hoechst $(\lambda$ exc $=364 \mathrm{~nm} ; \lambda$ em $=430-481 \mathrm{~nm})$, Alexa $488(\lambda$ exc $=495 \mathrm{~nm} ; \lambda \mathrm{em}$ $=500-600 \mathrm{~nm})$ and mScarlet $(\lambda \operatorname{exc}=561 \mathrm{~nm} ; \lambda \mathrm{em}=648-708 \mathrm{~nm})$. 


\section{Fluorescence spectroscopy}

Fluorescence spectra were obtained on SAFAS Xenius Spectrophotometer. Measurements were made in quartz cuvettes. A $1 \mathrm{~mL}$ solution was excited at $480 \mathrm{~nm}$. Solutions contained NES supplemented with $10 \mu \mathrm{M}$ YO-PRO-1 alone (-dsDNA in Fig. 4D), or $10 \mu \mathrm{M}$ YOPRO-1 complexed to $29 \mu \mathrm{g}$ dsDNA (+dsDNA), or $10 \mu \mathrm{M}$ YO-PRO-1 complexed to $29 \mu \mathrm{g}$ dsDNA with inhibitors (+dsDNA + inhibitors). Inhibitor concentrations used were the same as those used for electrophysiology. The bandwidth for excitation and emission was $10 \mathrm{~nm}$.

\section{Data analysis}

For electrophysiological data analysis, FitMaster (HEKA Electronics v2x73x1) and Igor Pro (WaveMetrics, v6.32A) were used. Experiments were repeated several times, over at least two independent transfections, with precise details given in figure legends. The extent of facilitation (as in Fig. 7A) was determined by calculating the fold difference between the $8^{\text {th }}$ agonist-evoked current and initial agonist-evoked current in a series of 8 repeated applications. The current density (as in Fig. 7A) was calculated as the $\mathrm{pA} / \mathrm{pF}$ of the $8^{\text {th }}$ agonist-evoked current in a series of 8 repeated applications.

For single channel data analysis, TAC and TACFit (Bruxton) were used. Data were refiltered offline to give a final $\mathrm{f}_{\mathrm{c}}$ of $100 \mathrm{~Hz}$. Dead time $\left(\mathrm{t}_{\mathrm{d}}\right)$ was set to $1.8 \mathrm{~ms}$, and transitions shorter than $t_{d}$ were ignored. Transitions longer than $t_{d}$ were accepted as events. Singlechannel current amplitudes were determined by all points histograms fitted to Gaussian distributions, using maximum likelihood methods:

$$
f(I)=\sum_{i=1}^{n} \frac{a_{i}}{\sigma_{i} \sqrt{2 \pi}} \exp \left[-\frac{\left(I-A_{i}\right)^{2}}{2 \sigma_{i}^{2}}\right]
$$

where $f(I)$ is the total probability density of a given amplitude value $I, A_{i}$ is the $i$ th channel amplitude, $\sigma_{i}$ is the standard deviation of the $i$ th channel amplitude, and $a_{i}$ is the fraction of the data represented by the $i$ th amplitude. Conductance was determined by dividing current amplitudes by the holding potential $(-120 \mathrm{mV})$.

For mean open time analysis, only patches featuring 3 or fewer channels were analyzed, and analysis of stacked events resulting from simultaneous channel openings was less than $5 \%$ of the total events analyzed. The number of channels present within a given patch can be determined by visual inspection of the maximum number of coinciding stacking events. These stacked openings are designated as such, in order to verify the proportion that they represent of the total events analyzed. For control condition, we recorded from 7 different outside-out patches, in which 1,518 events were analyzed. Each patch contained between 1 to 15 sweeps. For facilitated condition, we recorded from 5 outside-out patches, in which 754 events were analyzed (ranged between 3 to 19 sweeps). For MCD-treated cells, we recorded single-channel currents from 7 outside-out patches excised from cells that were pre-treated with MCD, and in which 396 events were analyzed (ranged between 1 to 13 sweeps). 
Detection of events was carried out by using $50 \%$ of the single-channel current amplitude as the detection threshold. Dwell time histograms were fitted by the minimum number of exponential functions according to the following equation:

$$
f(t)=\sum_{i=1}^{n} a_{i} / \tau_{i} \exp \left(-t / \tau_{i}\right)
$$

where $t$ is the time, $\tau_{i}$ is the time constant, and $a_{i}$ is the relative area (the sum of all areas is equal to 1). The mean open time for a selected state is given by:

$$
\tau_{\text {state }}=\sum a_{i} \tau_{i}
$$

For conductance analysis, the same patches were analyzed as in mean open time analysis, with the addition of supplementary patches. These supplementary patches contained too many stacked events to be considered for mean open time analysis, but remain suitable for unitary conductance analysis.

For analysis of percentage inhibition of $\mathrm{rP} 2 \mathrm{X} 7$ single-channel currents by $\mathrm{CaCC}$ inhibitors, a weighting is established of open-channel activity compared to closed-channel activity (within the 10s period of drug application, illustrated in Fig. 2C by grey shading). This weighting is calculated for recordings in patches exposed solely to BzATP, and then for subsequent recordings of the same patch exposed to BzATP and CaCC inhibitor coapplications. In the case of patches which did not exhibit instant inhibition, BzATP and $\mathrm{CaCC}$ inhibitor co-applications were repeated until a steady-state level of inhibition was achieved. These steady-state inhibitions were then averaged. A percentage inhibition is therefore calculated for each patch, which are subsequently averaged.

Deactivation currents (decay currents when BzATP is removed) were fit by single exponentials according to the following equation:

$$
f(t)=\mathrm{A} \exp \left(-t / \tau_{d}\right)
$$

where $t$ is the time, $\tau_{d}$ is time constant of deactivation, and $\mathrm{A}$ is the current amplitude.

For fluorescence experiment analysis, Fiji and Igor Pro were used. For co-localization analysis Pearson's correlation coefficient was measured using Coloc 2 in ImageJ.

\section{Molecular modeling}

The structures from P2X7 (6u9v and 6u9w) and TMEM16F (6qpc) were retrieved from the "Orientations of Proteins in Membranes (OPM) database" 54 . They are therefore oriented in the membrane with $\mathrm{x}$ and $\mathrm{y}$ being in the membrane plane and $\mathrm{z}$ being the normal to the plane and their axis of symmetry. P2X7 has been moved in the $\mathrm{x} / \mathrm{y}$ directions to separate it from TMEM16F. All structures were prepared for docking with the reduce command of ptools $^{55}$. Docking was performed with an inhouse python script that calls the ptools library ${ }^{56}$. The docking was performed in the membrane plane, i.e. the Monte-Carlo protocol 
explored the 3 relevant dimensions: rotation around the $\mathrm{z}$ axis or translation in $\mathrm{x}$ or $\mathrm{y}$ direction. After each random move in either of these dimensions the energy was evaluated with the Attract Force-Field and the resulting conformation was kept if the energy improved according to the force field. The relative orientations of the proteins were tested by generating 12 starting points through cumulative rotations of P2X7 by 10 degrees. Each starting point was tested 12 times.

\section{Statistical analysis}

Data are reported as mean values \pm SEM. All experiments were replicated over a minimum of 2 transfections and 3 cells. For electrophysiological data, exact data points are shown as dots within histograms, and for fluorescence data, the number of cells is shown in brackets. For statistical analysis, GraphPad Prism software (v8.0.2) was used. The normality of data distribution was tested before selection of an appropriate test of statistical significance and $P<0.05$ was considered as significant difference. For electrophysiological and Western blot data, Mann-Whitney or two-tailed (paired or unpaired, when indicated) Student's $t$ test was used. For fluorescence measurements, one-way Kruskal-Wallis analysis of variance test followed by Dunn's multiple comparison post-hoc test or Mann-Whitney test was employed, as indicated in figure legends.

\section{Data Availability}

All data and associated protocols for this study are available in the main text and SI Appendix. Materials may be requested to T.G.

\section{Acknowledgments}

We are grateful to F. Rassendren for providing WT tagged rP2X7 construct, R. Vauchelles for imaging advising, and D. Lemoine for help in imaging analysis. We are also grateful to L. Sachs for providing Axolotl oocytes (UMR 7221 PhyMA, CNRS and MNHN, Paris, France). This work was supported by the Agence Nationale de la Recherche (Grant ANR14-CE11-0004-01), the International Center for Frontier Research in Chemistry (Labex CSC-TGR-18), the labex Dynamo (ANR-11-LABX-0011), the Ministère de la Recherche, The Région Grand Est, and the "École Universitaire de Recherche" Euridol (Programme d'investissement d'Avenir). This work has also benefitted from support provided by the University of Strasbourg Institute for Advanced Study (USIAS) for a Fellowship (to F.A.P.), within the French national program "Investment for the future" (Idex-Unistra).

\section{Competing interests}

The authors declare no competing interests.

\section{References}

1 Habermacher, C., Dunning, K., Chataigneau, T. \& Grutter, T. Molecular structure and function of P2X receptors. Neuropharmacology 104, 18-30, doi:10.1016/j.neuropharm.2015.07.032 (2016). 
2 Volonte, C., Apolloni, S., Skaper, S. D. \& Burnstock, G. P2X7 receptors: channels, pores and more. CNS Neurol Disord Drug Targets 11, 705-721 (2012). 3 Surprenant, A., Rassendren, F., Kawashima, E., North, R. A. \& Buell, G. The cytolytic P2Z receptor for extracellular ATP identified as a $\mathrm{P} 2 \mathrm{X}$ receptor (P2X7). Science 272, 735-738, doi:10.1126/science.272.5262.735 (1996).

4 Roger, S., Pelegrin, P. \& Surprenant, A. Facilitation of P2X7 receptor currents and membrane blebbing via constitutive and dynamic calmodulin binding. J Neurosci $\mathbf{2 8}$, 6393-6401, doi:10.1523/JNEUROSCI.0696-08.2008 (2008).

5 Hibell, A. D., Kidd, E. J., Chessell, I. P., Humphrey, P. P. \& Michel, A. D. Apparent species differences in the kinetic properties of $\mathrm{P} 2 \mathrm{X}(7)$ receptors. $\mathrm{Br} J$ Pharmacol 130, 167-173, doi:10.1038/sj.bjp.0703302 (2000).

6 Robinson, L. E., Shridar, M., Smith, P. \& Murrell-Lagnado, R. D. Plasma membrane cholesterol as a regulator of human and rodent $\mathrm{P} 2 \mathrm{X} 7$ receptor activation and sensitization. J Biol Chem 289, 31983-31994, doi:10.1074/jbc.M114.574699 (2014). 7 Murrell-Lagnado, R. D. Regulation of P2X Purinergic Receptor Signaling by Cholesterol. Curr Top Membr 80, 211-232, doi:10.1016/bs.ctm.2017.05.004 (2017). 8 Karasawa, A., Michalski, K., Mikhelzon, P. \& Kawate, T. The P2X7 receptor forms a dye-permeable pore independent of its intracellular domain but dependent on membrane lipid composition. Elife 6, doi:10.7554/eLife.31186 (2017).

9 Di Virgilio, F., Schmalzing, G. \& Markwardt, F. The Elusive P2X7 Macropore. Trends Cell Biol, doi:10.1016/j.tcb.2018.01.005 (2018).

10 Steinberg, T. H., Newman, A. S., Swanson, J. A. \& Silverstein, S. C. ATP4permeabilizes the plasma membrane of mouse macrophages to fluorescent dyes. J Biol Chem 262, 8884-8888 (1987).

11 Cockcroft, S. \& Gomperts, B. D. ATP induces nucleotide permeability in rat mast cells. Nature 279, 541-542, doi:10.1038/279541a0 (1979).

12 Jindrichova, M. et al. Functional characterization of mutants in the transmembrane domains of the rat $\mathrm{P} 2 \mathrm{X} 7$ receptor that regulate pore conductivity and agonist sensitivity. J Neurochem 133, 815-827, doi:10.1111/jnc.13078 (2015). 13 Yan, Z., Li, S., Liang, Z., Tomic, M. \& Stojilkovic, S. S. The P2X7 receptor channel pore dilates under physiological ion conditions. J Gen Physiol 132, 563-573, doi:10.1085/jgp.200810059 (2008).

14 Pelegrin, P. \& Surprenant, A. Pannexin-1 mediates large pore formation and interleukin-1beta release by the ATP-gated P2X7 receptor. EMBO J 25, 5071-5082, doi:10.1038/sj.emboj.7601378 (2006).

15 Virginio, C., MacKenzie, A., Rassendren, F. A., North, R. A. \& Surprenant, A. Pore dilation of neuronal P2X receptor channels. Nature neuroscience 2, 315-321, doi:10.1038/7225 (1999). 16 Khakh, B. S., Bao, X. R., Labarca, C. \& Lester, H. A. Neuronal P2X transmittergated cation channels change their ion selectivity in seconds. Nature neuroscience 2, 322330, doi:10.1038/7233 (1999).

17 Li, M., Toombes, G. E., Silberberg, S. D. \& Swartz, K. J. Physical basis of apparent pore dilation of ATP-activated P2X receptor channels. Nat Neurosci 18, 15771583, doi:10.1038/nn.4120 (2015). 
18 Riedel, T., Schmalzing, G. \& Markwardt, F. Influence of extracellular monovalent cations on pore and gating properties of $\mathrm{P} 2 \mathrm{X} 7$ receptor-operated singlechannel currents. Biophys J 93, 846-858, doi:10.1529/biophysj.106.103614 (2007). 19 Pippel, A. et al. Localization of the gate and selectivity filter of the full-length P2X7 receptor. Proc Natl Acad Sci U S A 114, E2156-E2165, doi:10.1073/pnas.1610414114 (2017).

20 Harkat, M. et al. On the permeation of large organic cations through the pore of ATP-gated P2X receptors. Proc Natl Acad Sci U S A 114, E3786-E3795, doi:10.1073/pnas.1701379114 (2017).

21 Peverini, L., Beudez, J., Dunning, K., Chataigneau, T. \& Grutter, T. New Insights Into Permeation of Large Cations Through ATP-Gated P2X Receptors. Front Mol Neurosci 11, 265, doi:10.3389/fnmol.2018.00265 (2018).

22 Janks, L., Sprague, R. S. \& Egan, T. M. ATP-Gated P2X7 Receptors Require Chloride Channels To Promote Inflammation in Human Macrophages. J Immunol 202, 883-898, doi:10.4049/jimmunol.1801101 (2019).

23 Ugur, M. \& Ugur, O. A Mechanism-Based Approach to P2X7 Receptor Action. Mol Pharmacol 95, 442-450, doi:10.1124/mol.118.115022 (2019).

24 Browne, L. E., Compan, V., Bragg, L. \& North, R. A. P2X7 receptor channels allow direct permeation of nanometer-sized dyes. J Neurosci 33, 3557-3566, doi:10.1523/JNEUROSCI.2235-12.2013 (2013).

25 McCarthy, A. E., Yoshioka, C. \& Mansoor, S. E. Full-Length P2X7 Structures

Reveal How Palmitoylation Prevents Channel Desensitization. Cell 179, 659-670 e613, doi:10.1016/j.cell.2019.09.017 (2019).

26 Ousingsawat, J. et al. Anoctamin 6 mediates effects essential for innate immunity downstream of P2X7 receptors in macrophages. Nat Commun 6, 6245, doi:10.1038/ncomms7245 (2015).

27 Janks, L., Sharma, C. V. R. \& Egan, T. M. A central role for P2X7 receptors in human microglia. J Neuroinflammation 15, 325, doi:10.1186/s12974-018-1353-8 (2018). 28 Stolz, M. et al. Homodimeric anoctamin-1, but not homodimeric anoctamin-6, is activated by calcium increases mediated by the P2Y1 and P2X7 receptors. Pflugers Arch 467, 2121-2140, doi:10.1007/s00424-015-1687-3 (2015).

29 Falzone, M. E., Malvezzi, M., Lee, B. C. \& Accardi, A. Known structures and unknown mechanisms of TMEM16 scramblases and channels. J Gen Physiol 150, 933947, doi:10.1085/jgp.201711957 (2018).

30 Norenberg, W. et al. TRPM7 is a molecular substrate of ATP-evoked P2X7-like currents in tumor cells. J Gen Physiol 147, 467-483, doi:10.1085/jgp.201611595 (2016). 31 Virginio, C., Church, D., North, R. A. \& Surprenant, A. Effects of divalent cations, protons and calmidazolium at the rat P2X7 receptor. Neuropharmacology 36, 1285-1294, doi:10.1016/s0028-3908(97)00141-x (1997).

32 Yan, Z. et al. Experimental characterization and mathematical modeling of P2X7 receptor channel gating. J Neurosci 30, 14213-14224, doi:10.1523/JNEUROSCI.239010.2010 (2010).

33 Riedel, T., Lozinsky, I., Schmalzing, G. \& Markwardt, F. Kinetics of P2X7 receptor-operated single channels currents. Biophys $J$ 92, 2377-2391, doi:10.1529/biophysj.106.091413 (2007). 
34 Namkung, W., Thiagarajah, J. R., Phuan, P. W. \& Verkman, A. S. Inhibition of $\mathrm{Ca} 2+$-activated $\mathrm{Cl}$ - channels by gallotannins as a possible molecular basis for health benefits of red wine and green tea. FASEB J 24, 4178-4186, doi:10.1096/fj.10-160648 (2010).

35 Liu, Y. et al. Characterization of the effects of $\mathrm{Cl}(-)$ channel modulators on TMEM16A and bestrophin-1 $\mathrm{Ca}(2)(+)$ activated $\mathrm{Cl}(-)$ channels. Pflugers Arch 467, $1417-$ 1430, doi:10.1007/s00424-014-1572-5 (2015).

36 Cherian, O. L., Menini, A. \& Boccaccio, A. Multiple effects of anthracene-9carboxylic acid on the TMEM16B/anoctamin2 calcium-activated chloride channel. Biochim Biophys Acta 1848, 1005-1013, doi:10.1016/j.bbamem.2015.01.009 (2015). 37 Schroeder, B. C., Cheng, T., Jan, Y. N. \& Jan, L. Y. Expression cloning of TMEM16A as a calcium-activated chloride channel subunit. Cell 134, 1019-1029, doi:10.1016/j.cell.2008.09.003 (2008).

38 Wozniak, K. L., Phelps, W. A., Tembo, M., Lee, M. T. \& Carlson, A. E. The TMEM16A channel mediates the fast polyspermy block in Xenopus laevis. J Gen Physiol 150, 1249-1259, doi:10.1085/jgp.201812071 (2018).

39 Alvadia, C. et al. Cryo-EM structures and functional characterization of the murine lipid scramblase TMEM16F. Elife 8, doi:10.7554/eLife.44365 (2019).

40 Yang, H. et al. TMEM16F forms a Ca2+-activated cation channel required for lipid scrambling in platelets during blood coagulation. Cell 151, 111-122, doi:10.1016/j.cell.2012.07.036 (2012).

41 Le, T., Le, S. C., Zhang, Y., Liang, P. \& Yang, H. Evidence that polyphenols do not inhibit the phospholipid scramblase TMEM16F. J Biol Chem, doi:10.1074/jbc.AC120.014872 (2020). 42 Shrivastava, A. N., Rodriguez, P. C., Triller, A. \& Renner, M. Dynamic microorganization of $\mathrm{P} 2 \mathrm{X} 7$ receptors revealed by PALM based single particle tracking. Front Cell Neurosci 7, 232, doi:10.3389/fncel.2013.00232 (2013).

43 Cankurtaran-Sayar, S., Sayar, K. \& Ugur, M. P2X7 receptor activates multiple selective dye-permeation pathways in RAW 264.7 and human embryonic kidney 293 cells. Mol Pharmacol 76, 1323-1332, doi:10.1124/mol.109.059923 (2009).

44 Ye, W., Han, T. W., He, M., Jan, Y. N. \& Jan, L. Y. Dynamic change of electrostatic field in TMEM16F permeation pathway shifts its ion selectivity. Elife 8 , doi:10.7554/eLife.45187 (2019).

$45 \mathrm{Yu}, \mathrm{K}$. et al. Identification of a lipid scrambling domain in ANO6/TMEM16F. Elife 4, e06901, doi:10.7554/eLife.06901 (2015).

46 Salm, E. J. et al. TMEM163 Regulates ATP-Gated P2X Receptor and Behavior. Cell Rep 31, 107704, doi:10.1016/j.celrep.2020.107704 (2020).

47 Di Virgilio, F., Dal Ben, D., Sarti, A. C., Giuliani, A. L. \& Falzoni, S. The P2X7 Receptor in Infection and Inflammation. Immunity 47, 15-31, doi:10.1016/j.immuni.2017.06.020 (2017).

48 Sorge, R. E. et al. Genetically determined P2X7 receptor pore formation regulates variability in chronic pain sensitivity. Nat Med 18, 595-599, doi:10.1038/nm.2710 (2012).

49 Batti, L. et al. TMEM16F Regulates Spinal Microglial Function in Neuropathic Pain States. Cell Rep 15, 2608-2615, doi:10.1016/j.celrep.2016.05.039 (2016). 
$98050 \quad$ Bertin, E. et al. Increased surface P2X4 receptor regulates anxiety and memory in 981 P2X4 internalization-defective knock-in mice. Mol Psychiatry, doi:10.1038/s41380-019982 0641-8 (2020).

$98351 \quad$ Pougnet, J. T. et al. P2X-mediated AMPA receptor internalization and synaptic 984 depression is controlled by two CaMKII phosphorylation sites on GluA1 in hippocampal 985 neurons. Sci Rep 6, 31836, doi:10.1038/srep31836 (2016).

$98652 \quad$ Le, T., Le, S. C. \& Yang, H. Drosophila Subdued is a moonlighting 987 transmembrane protein 16 (TMEM16) that transports ions and phospholipids. $J$ Biol 988 Chem 294, 4529-4537, doi:10.1074/jbc.AC118.006530 (2019).

98953 Ran, F. A. et al. Genome engineering using the CRISPR-Cas9 system. Nat Protoc 990 8, 2281-2308, doi:10.1038/nprot.2013.143 (2013).

99154 Lomize, M. A., Pogozheva, I. D., Joo, H., Mosberg, H. I. \& Lomize, A. L. OPM 992 database and PPM web server: resources for positioning of proteins in membranes.

$993 \quad$ Nucleic Acids Res 40, D370-376, doi:10.1093/nar/gkr703 (2012).

$99455 \quad$ Schneider, S., Saladin, A., Fiorucci, S., Prevost, C. \& Zacharias, M. ATTRACT 995 and PTools: open source programs for protein-protein docking. Methods Mol Biol 819, 996 221-232, doi:10.1007/978-1-61779-465-0_15 (2012).

$99756 \quad$ Saladin, A., Fiorucci, S., Poulain, P., Prevost, C. \& Zacharias, M. PTools: an 998 opensource molecular docking library. BMC Struct Biol 9, 27, doi:10.1186/1472-6807-9$99927(2009)$.

1000

1001 
A
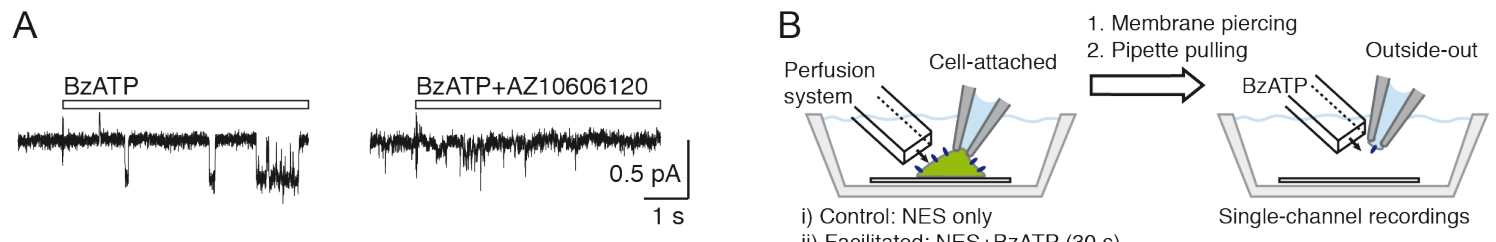

ii) Facilitated: NES+BzATP (30 s)

iii) MCD: NES following MCD incubation
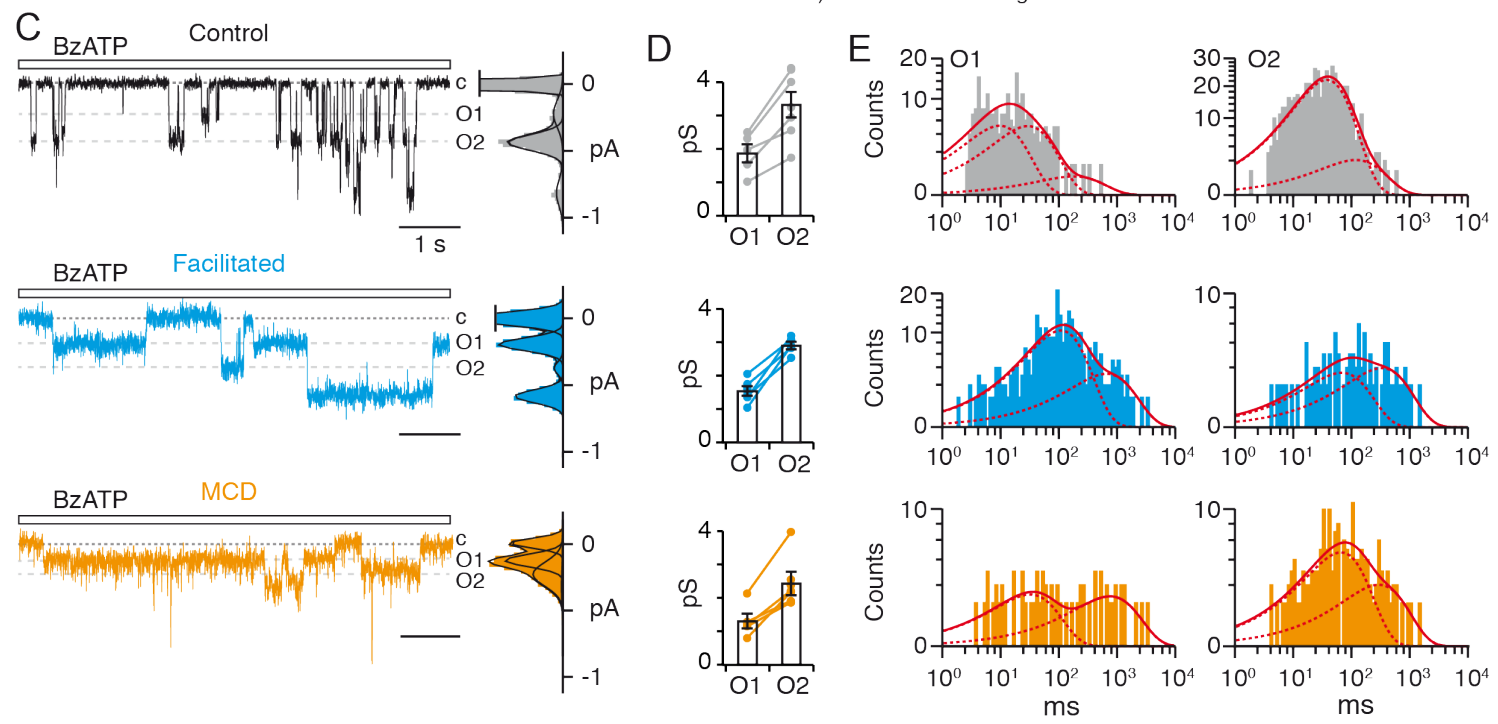

Figure 1. Current facilitation of $\mathrm{P} 2 \mathrm{X} 7$ results in an increase of single-channel open probability. (A) Single-channel currents elicited by $10 \mu \mathrm{M}$ BzATP (duration of 1007 application indicated by the white bar) from an outside-out patch of HEK293T cell transiently transfected with $\mathrm{rP} 2 \mathrm{X} 7$ (left) are inhibited by $1 \mu \mathrm{M}$ AZ10606120 when coapplied with $10 \mu \mathrm{M}$ BzATP (right). Traces are from the same patch and channel openings are downward deflections. (B) Cartoon depicting the protocol employed for establishing excised patches from cells expressing rP2X7 (blue ovals) in: i) control conditions, ii) facilitated conditions (10 $\mathrm{MM}$ BzATP for $30 \mathrm{~s}$ ), and iii) MCD-treated conditions. NES: Normal extracellular solution. Figure not to scale. (C) Single-channel currents elicited by $10 \mu \mathrm{M}$ BzATP from outside-out patches of HEK293T cells transiently transfected with rP2X7. Cells were either not treated (black), or pre-treated with a $30 \mathrm{~s}$ perfusion of $10 \mu \mathrm{M}$ BzATP (blue), or incubated with MCD (orange) prior to membrane excising. Dashed grey lines indicate unitary currents corresponding to conductance states $\mathrm{O} 1$ and $\mathrm{O} 2$. Dotted lines indicate basal current corresponding to closed channels, labeled c. Corresponding all points histograms of currents are shown right of the traces. Distributions were fit by a sum of Gaussians. (D) Summary of corresponding unitary conductances. Linking lines indicate data points that originate from the same patches $(n=7$ patches for control, 6 for facilitated and 7 for MCD-treated conditions). Bars represent mean \pm SEM. (E) Corresponding open dwell-time histograms fitted by the sum (solid red line) of several exponential functions (dashed red lines). For control patches (grey histograms), $\tau_{1}=9.6 \mathrm{~ms}$, relative area, $\mathrm{a}_{1}=$ $0.49 ; \tau_{2}=29 \mathrm{~ms}, \mathrm{a}_{2}=0.48 ; \tau_{3}=180 \mathrm{~ms}, \mathrm{a}_{3}=0.03$; mean open time, $\tau_{\mathrm{O} 1}=24 \mathrm{~ms}$ for O1, and $\tau_{1}=38 \mathrm{~ms}, \mathrm{a}_{1}=0.92 ; \tau_{2}=119 \mathrm{~ms}, \mathrm{a}_{2}=0.08 ; \tau_{\mathrm{O} 2}=44 \mathrm{~ms}$ for $\mathrm{O} 2$. For facilitated (blue 
1027 histograms), $\tau_{1}=108 \mathrm{~ms}, \mathrm{a}_{1}=0.77 ; \tau_{2}=654 \mathrm{~ms}, \mathrm{a}_{2}=0.23 ; \tau_{\mathrm{O} 1}=234 \mathrm{~ms}$ for $\mathrm{O} 1$ and $\tau_{1}=68$ $1028 \mathrm{~ms}, \mathrm{a}_{1}=0.46 ; \tau_{2}=332 \mathrm{~ms}, \mathrm{a}_{2}=0.54 ; \tau_{\mathrm{O} 2}=211 \mathrm{~ms}$ for O2. For MCD-treated patches (orange 1029 histograms), $\tau_{1}=32 \mathrm{~ms}, \mathrm{a}_{1}=0.52 ; \tau_{2}=752 \mathrm{~ms}, \mathrm{a}_{2}=0.48 ; \tau_{\mathrm{O} 1}=378 \mathrm{~ms}$, for $\mathrm{O} 1$ and $\tau_{1}=66$ $1030 \mathrm{~ms}, \mathrm{a}_{1}=0.70 ; \tau_{2}=294 \mathrm{~ms}, \mathrm{a}_{2}=0.30 ; \tau_{\mathrm{O} 2}=134 \mathrm{~ms}$ for $\mathrm{O} 2$. All data were recorded at -120 $1031 \mathrm{mV}$ and filtered at a final $\mathrm{f}_{\mathrm{c}}$ of $100 \mathrm{~Hz}$. 

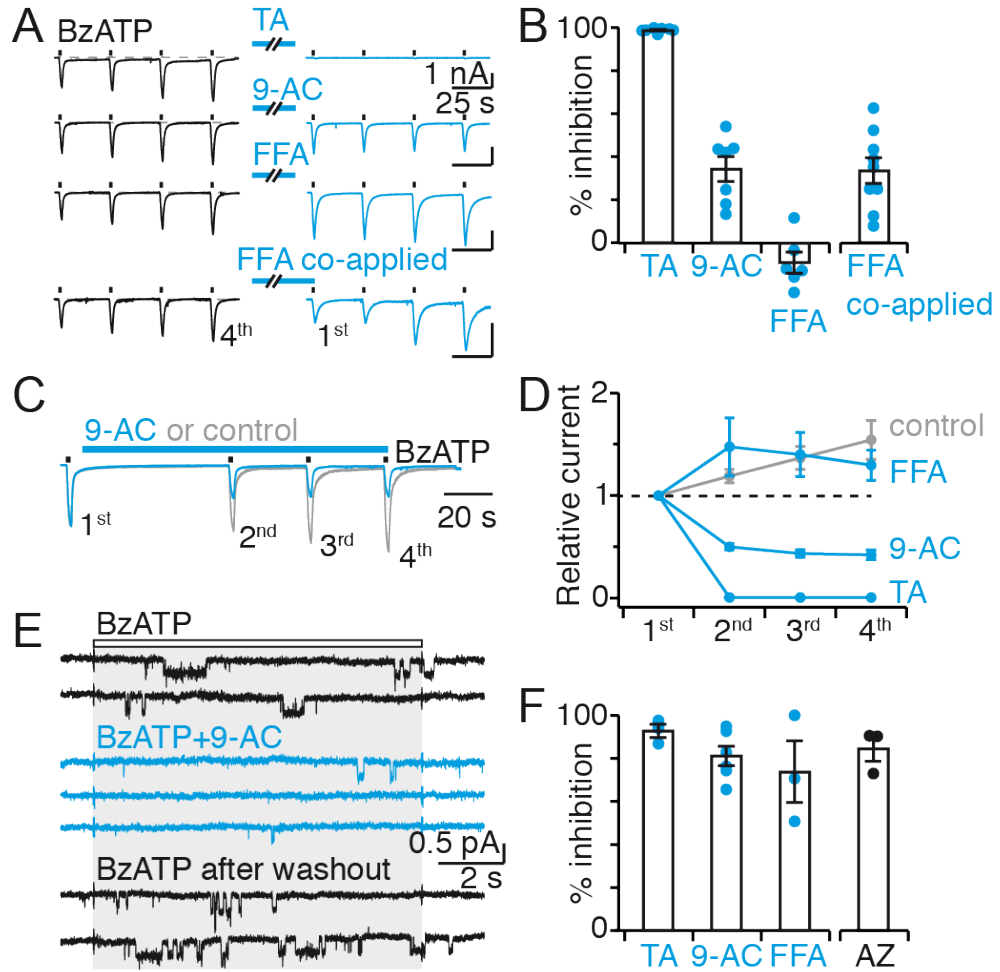

1033

1034

1035

1036

1037

1038

1039

1040

1041

1042

1043

1044

1045

1046

1047

1048

1049

1050

1051

1052

1053

1054
Figure 2. CaCC inhibitors inhibit P2X7 currents in HEK293T cells. (A) Whole-cell currents evoked by repeated $2 \mathrm{~s}$ applications of $10 \mu \mathrm{M}$ BzATP (black bars) before and after 60 s perfusion of TMEM16 inhibitors (blue bars) in HEK293T cells transiently expressing rP2X7. In the case of FFA co-applied, perfusion was followed by a co-application with 10 $\mu \mathrm{M}$ BzATP. (B) Summary of whole-cell inhibition ( $\mathrm{n}=7$ cells for TA, 7 for 9-AC, 6 for FFA, and 9 for FFA co-applied). Inhibition is calculated by comparing current amplitude of the fourth application before and first application after perfusion. Bars represent mean \pm SEM. (C) Whole-cell currents evoked by repeated $2 \mathrm{~s}$ applications of $10 \mu \mathrm{M}$ BzATP in the presence (blue) or absence of 9-AC (grey) in HEK293T cells transiently expressing rP2X7. Currents are normalized to the first application. (D) Summary of currents relative to the first application in the absence (control) or presence of inhibitors ( $\mathrm{n}=4$ cells for TA, 10 for 9 -AC, 8 for FFA, and 8 for control). Symbols represent mean \pm SEM. (E) Singlechannel rP2X7 currents elicited by $10 \mu \mathrm{M}$ BzATP from an outside-out patch of HEK293T cell (black traces) are inhibited by $1 \mathrm{mM}$ 9-AC when co-applied with $10 \mu \mathrm{M}$ BzATP (blue traces). Shown sweeps were sequentially recorded from the same patch. Note that inhibition is reversible after washout. Duration of application is indicated by the white bar and grey shading. Data were filtered at a final $f_{c}$ of $100 \mathrm{~Hz}$. (F) Summary of single-channel inhibition ( $\mathrm{n}=3$ patches for TA, 6 for 9-AC, 3 for FFA, and 3 for AZ10606120). Bars represent mean \pm SEM. See Materials and Methods for calculation. Inhibitor concentrations were $20 \mu \mathrm{M}$ TA, $1 \mathrm{mM}$ 9-AC, $100 \mu \mathrm{M}$ FFA and $1 \mu \mathrm{M}$ AZ10606120. Singlechannel and whole-cell data were recorded at -120 and $-60 \mathrm{mV}$, respectively. 
A Axolotl oocytes

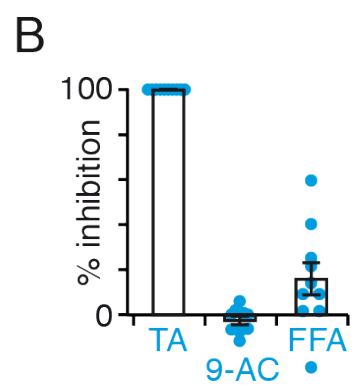

C

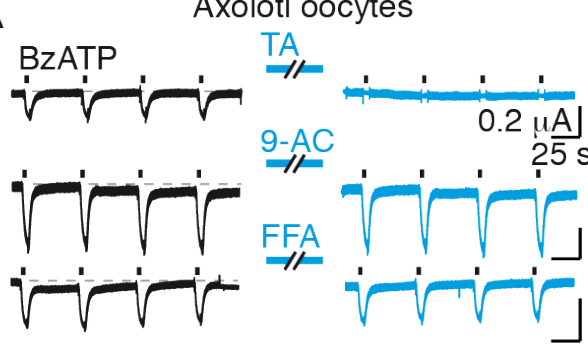

Xenopus oocytes

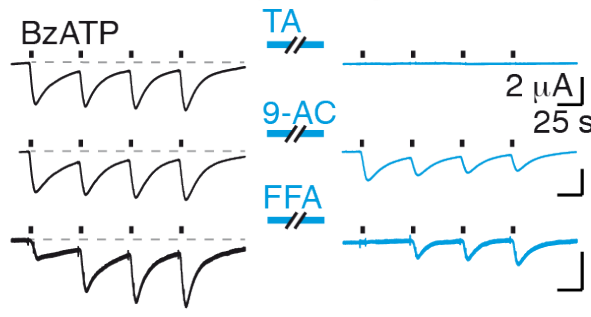

$\mathrm{D}$

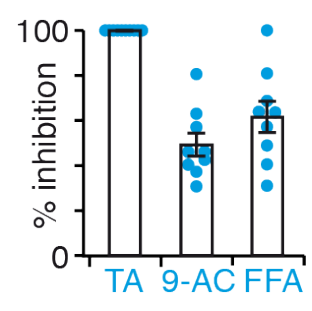

Figure 3. 9-AC and FFA do not inhibit P2X7 currents in Axolotl. (A) Whole-cell and after application of TMEM16 inhibitors using the same protocol as in Fig. 2A. (B) Summary of inhibition in Axolotl oocytes calculated as in Fig. $2 \mathrm{~B}(\mathrm{n}=10$ oocytes for TA, 9 for 9 -AC, and 10 for FFA). Bars represent mean \pm SEM. (C) Whole-cell BzATP-evoked currents recorded by TEVC of Xenopus oocytes expressing rP2X7 using the same protocol as in Fig. 2A. (D) Summary of inhibition in Xenopus oocytes calculated as in Fig. 2B ( $\mathrm{n}=$ 10 oocytes for TA, 9 for $9-\mathrm{AC}$, and 9 for FFA). Bars represent mean \pm SEM. CaCC inhibitor concentrations were $20 \mu \mathrm{M}$ TA, $1 \mathrm{mM}$ 9-AC and $100 \mu \mathrm{M}$ FFA. Data were recorded at $-60 \mathrm{mV}$. 

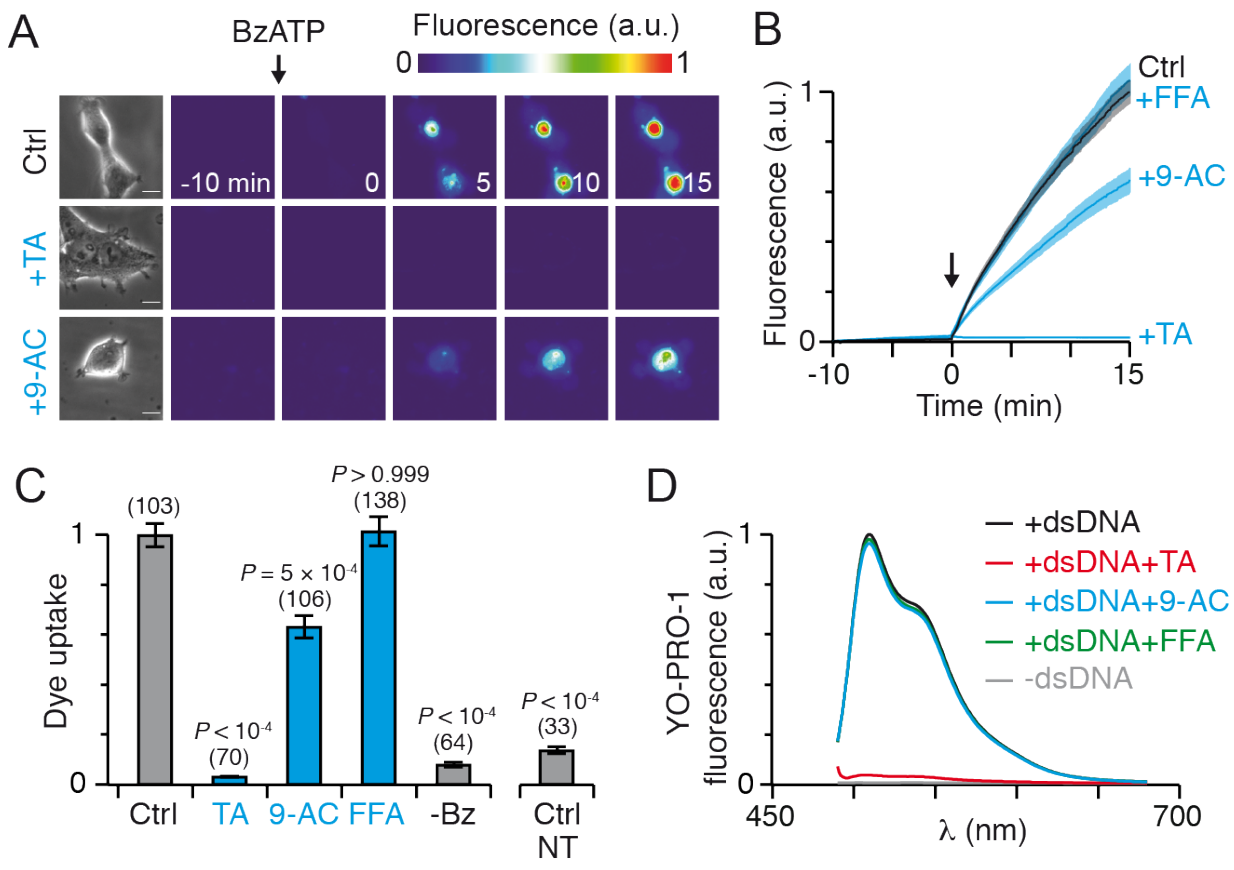

1069

1070

1071

1072

1073

1074

1075

1076

1077

1078

1079

1080

1081

1082

1083

1084

1085

1086

1087

1088

1089

Figure 4. Effects of CaCC inhibitors on dye uptake. (A) Time series fluorescence images of YO-PRO-1 dye uptake in HEK293T cells transiently expressing rP2X7, in the absence or presence of inhibitors, as indicated. Fluorescence (in arbitrary units) was acquired at indicated times before and after $10 \mu \mathrm{M}$ BzATP (arrow) application, which begins at $0 \mathrm{~min}$. Inhibitors were co-applied at the same time as BzATP. On the left are shown the corresponding microphotographs under transmitted light (scale bar, $10 \mu \mathrm{m}$ ). (B) Corresponding YO-PRO-1 dye uptake over time upon BzATP activation (arrow) in the absence or presence of inhibitors, as indicated. Solid lines represent mean \pm SEM (shaded areas). (C) Summary of BzATP-induced YO-PRO-1 dye uptake normalized to rP2X7transfected cells in the absence (Ctrl, grey histogram) or presence of inhibitors (blue histograms). Numbers of cells are indicated above bars. Dye uptake in the absence of BzATP is labelled -Bz. BzATP-induced dye uptake in non-transfected cells (NT) was normalized to another set of $\mathrm{rP} 2 \mathrm{X} 7$-transfected control $(\mathrm{n}=78$ cells). Bars represent mean \pm SEM (from 3 to 12 transfections). $P$ values are from one-way Kruskal-Wallis ANOVA followed by Dunn's comparison to respective controls. (D) Fluorescence spectra of $10 \mu \mathrm{M}$ YO-PRO-1 bound to double strand DNA (dsDNA) recorded before (black trace, averaged from respective controls) and immediately after application of TA (red), 9-AC (blue) or FFA (green). Fluorescence spectra of $10 \mu \mathrm{M}$ unbound YO-PRO-1 (in the absence of dsDNA) is indicated in grey. CaCC inhibitor concentrations were $20 \mu \mathrm{M} \mathrm{TA}, 1 \mathrm{mM}$ 9-AC and $100 \mu \mathrm{M}$ FFA. 

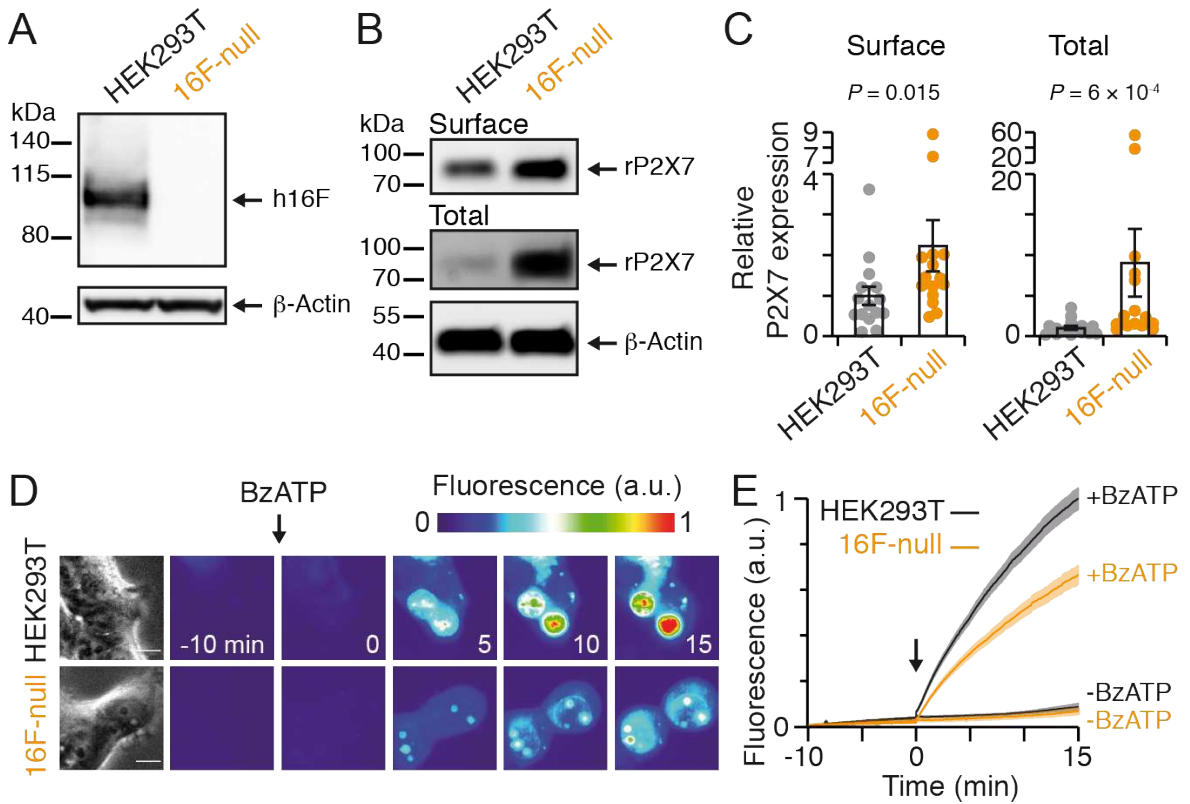

F

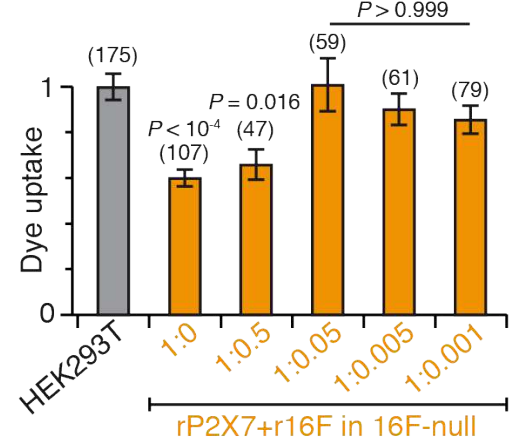

G

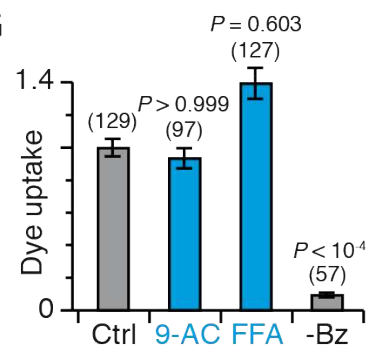

Figure 5. TMEM16F contributes to dye uptake. (A) Western blot analysis of total cell lysate separated by SDS-PAGE indicates the successful knock-out of endogenous hTMEM16F (h16F) by CRISPR/Cas9 in 16F-null cells. Western blotting was performed with anti-hTMEM16F antibody. $\beta$-Actin was used as a loading control. (B) Total and surface expression of myc tagged rP2X7 subunits transiently expressed in HEK293T or in 16F-null cells and resolved by SDS-PAGE. Proteins at the cell surface were biotinylated and isolated with neutravidin-bound agarose resin. Western blotting was performed with anti-c-myc antibody. $\beta$-Actin was used as a loading control. Apparent molecular weight markers are indicated. Uncropped blots are shown in Fig. S6A and B. (C) Summary of relative $\mathrm{P} 2 \mathrm{X} 7$ expression normalized to $\beta$-actin. Bars represent mean \pm SEM (from 15 transfections). $P$ values are from Mann-Whitney test. (D) Time series fluorescence images of YO-PRO-1 dye uptake upon $10 \mu \mathrm{M}$ BzATP activation (arrow) in HEK293T cells and 16F-null cells transiently expressing rP2X7. See legend of Fig. 4A for details. Scale bar, $10 \mu \mathrm{m}$. (E) Corresponding YO-PRO-1 uptake over time in HEK293T cells (black) and $16 \mathrm{~F}$-null cells (orange), in the absence (-BzATP) or presence of $10 \mu \mathrm{M}$ BzATP (+BzATP) applied as indicated (arrow). Solid lines represent mean \pm SEM (shaded areas). (F) Summary of BzATP-induced YO-PRO-1 uptake in 16F-null cells transiently expressing 
$1108 \mathrm{rP} 2 \mathrm{X} 7(2 \mu \mathrm{g})$ in the absence (ratio 1:0) or presence of rTMEM16F, transfected with varying 1109 quantities of cDNA (from 1 to $0.002 \mu \mathrm{g}$ ) at the indicated ratios (defined as $\mathrm{rP} 2 \mathrm{X} 7$ to 1110 TMEM16F). Shown is the specific dye uptake, found by subtracting residual uptake 1111 recorded in the absence of BzATP. Data are normalized to rP2X7-expressing HEK293T 1112 cells. Bars represent mean \pm SEM, and numbers of cells are indicated above bars (from 4 1113 to 5 transfections). $P$ values are from one-way Kruskal-Wallis ANOVA followed by 1114 Dunn's comparison to HEK293T. (G) Summary of YO-PRO-1 uptake in 16F-null cells in 1115 the absence (Ctrl) or presence of TMEM16 inhibitors. Dye uptake in the absence of BzATP 1116 is labeled $-\mathrm{Bz}$. Bars represent mean \pm SEM, and numbers of cells are indicated above bars 1117 (from 5 transfections). $P$ values are from one-way Kruskal-Wallis ANOVA followed by 1118 Dunn's comparison to control (Ctrl). CaCC inhibitor concentrations were $1 \mathrm{mM}$ 9-AC and $1119100 \mu \mathrm{M}$ FFA. 

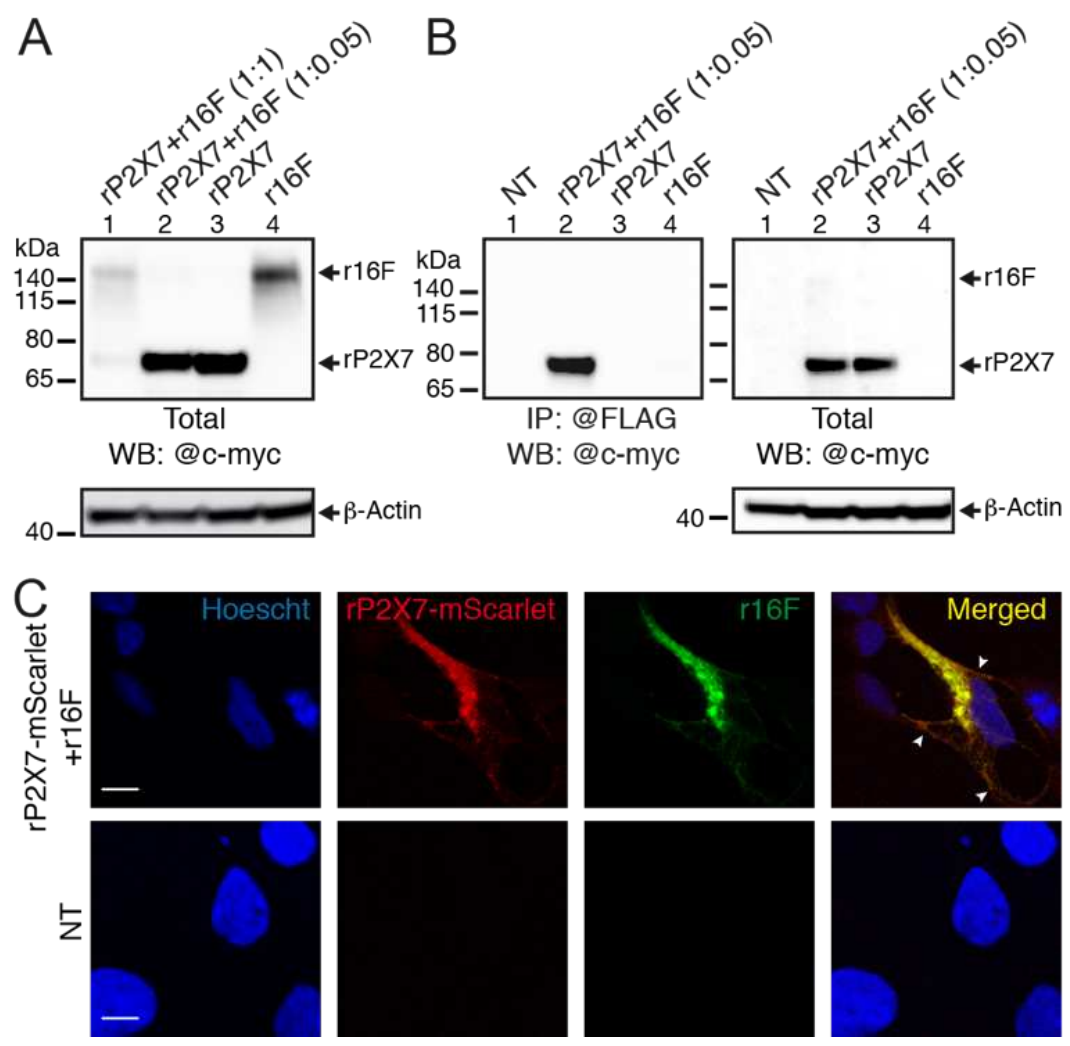

Figure 6. Close physical proximity of P2X7 and TMEM16F. (A) Western blot of total cell lysate separated by SDS-PAGE from 16F-null cells transiently transfected with myc tagged rP2X7 (rP2X7) and myc-DDK tagged rTMEM16F (r16F) at a ratio of 1:1 (lane 1: $5 \mu \mathrm{g} \mathrm{rP} 2 \mathrm{X} 7$ and $5 \mu \mathrm{g} \mathrm{r} 16 \mathrm{~F}$ ) or 1:0.05 (lane 2: $5 \mu \mathrm{g} \mathrm{rP} 2 \mathrm{X} 7$ and $0.25 \mu \mathrm{g} \mathrm{r16F}$ ) and probed with anti-c-myc antibody. Note reduced $\mathrm{rP} 2 \mathrm{X} 7$ subunit expression in lane 1 compared to that in lane 2. Control expressions of $\mathrm{rP} 2 \mathrm{X} 7(10 \mu \mathrm{g})$ and $\mathrm{r} 16 \mathrm{~F}(10 \mu \mathrm{g})$ are shown in lane 3 and 4 , respectively. $\beta$-Actin was used as a loading control. Apparent molecular weight markers are indicated. (B) Left: Co-immunoprecipitation reveals physical association of myc tagged $\mathrm{rP} 2 \mathrm{X} 7$ and myc-DDK tagged $\mathrm{r} 16 \mathrm{~F}$ subunits transiently co-expressed at a ratio of 1:0.05 (5 $\mu \mathrm{g} \mathrm{rP} 2 \mathrm{X} 7$ and $0.25 \mu \mathrm{g}$ r16F) in 16F-null cells and resolved by SDS-PAGE. Co-immunoprecipitation was performed in cell lysate with anti-FLAG antibody, which recognizes DDK tag of myc-DDK tagged $\mathrm{r} 16 \mathrm{~F}$, and Western blotting was performed with anti-c-myc antibody. Right: Western blot of total cell lysate of corresponding samples probed with anti-c-myc antibody. Control expressions of rP2X7 $(5 \mu \mathrm{g})$ and r16F $(0.25 \mu \mathrm{g})$ are shown in lanes 3 and 4 , respectively. Note that the band corresponding to myc-DDK tagged $\mathrm{r} 16 \mathrm{~F}$ is barely visualized because of the 20 -fold lower expression of myc-DDK tagged r16F compared to that of $\mathrm{rP} 2 \mathrm{X} 7$ (see, however, Fig. S6D for the actual presence of $\mathrm{r} 16 \mathrm{~F})$. Controls in non-transfected (NT) cells are shown in lanes $1 . \beta$-Actin is used as the loading control. Uncropped blots are shown in Fig. S6C and D. (C) Top: Confocal images of 16F-null cells transiently transfected with $2 \mu \mathrm{g}$ rP2X7-mScarlet (red) and $0.1 \mu \mathrm{g}$ r16F (green) demonstrate co-localization (yellow) of both proteins. Head arrows indicate putative cell membrane localizations. Cells were stained with a primary anti-h16F antibody 
1143 coupled to a secondary Alexa 488 conjugated antibody (green). Nuclei were stained with 1144 Hoescht (blue). Bottom: Control in non-transfected (NT) 16F-null cells. Scale bar, $10 \mu \mathrm{m}$. 
1146

1147

1148

1149

1150

1151

1152

1153

1154

1155

1156

1157

1158

1159

1160

1161

1162

1163

1164

1165

1166

1167

1168

1169

1170

1171

1172

1173
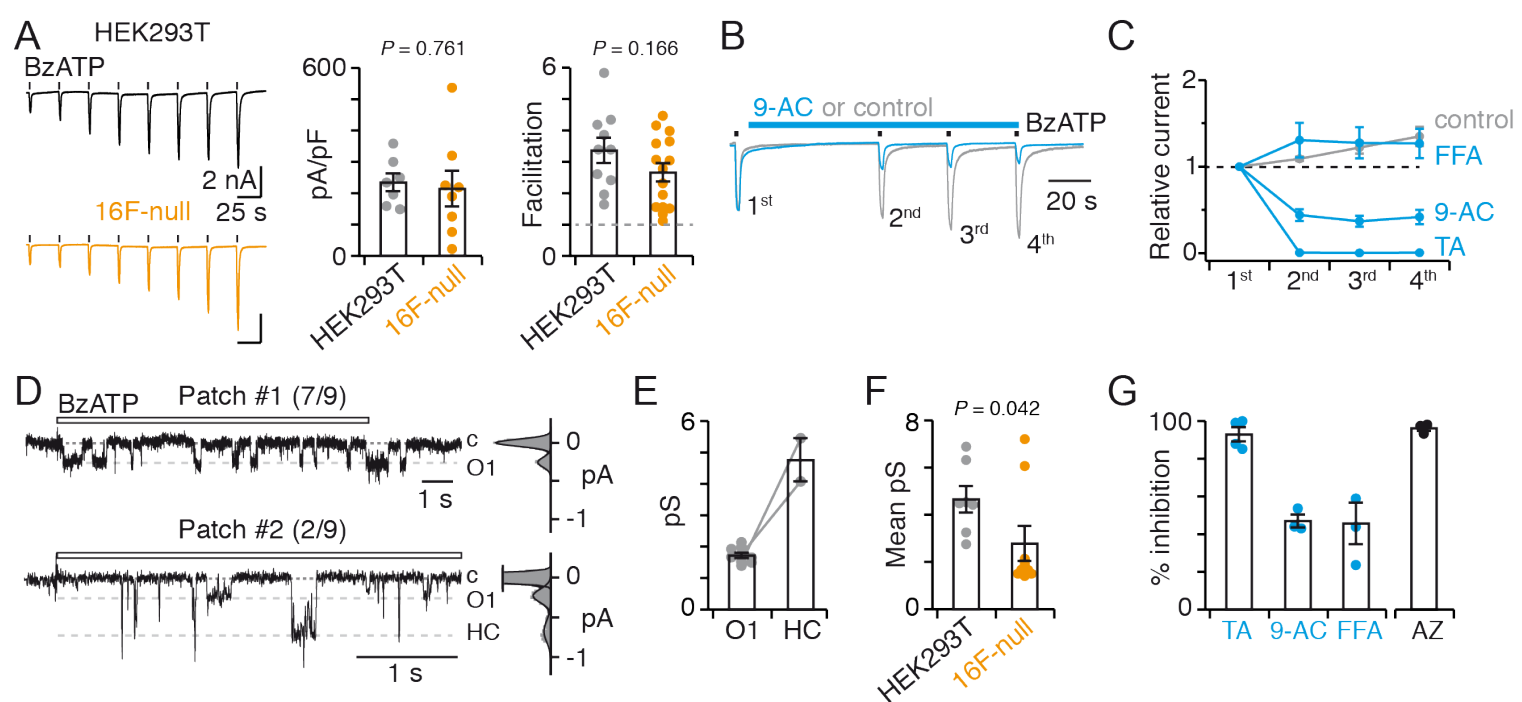

Figure 7. TMEM16F shapes P2X7 unitary conductance. (A) Left: whole-cell currents evoked by repeated $2 \mathrm{~s}$ applications of $10 \mu \mathrm{M}$ BzATP recorded in HEK293T (black trace) and 16F-null cells (orange trace) transiently transfected with rP2X7. Middle: summary of current density of the $8^{\text {th }}$ application ( $\mathrm{n}=7$ for HEK293T cells and 8 for $16 \mathrm{~F}$-null cells). Bars represent mean \pm SEM. Right: summary of current facilitation determined between the $8^{\text {th }}$ and $1^{\text {st }}$ application. See Materials and Methods for calculation $(n=10$ cells for HEK293T and 15 for 16F-null). $P$ values are from unpaired Student's $t$-test. (B) Wholecell currents evoked by repeated $2 \mathrm{~s}$ applications of $10 \mu \mathrm{M}$ BzATP in the presence (blue) or absence of 9-AC (grey) in 16F-null cells transiently expressing rP2X7. Currents are normalized to the first application. (C) Summary currents relative to the first application in the absence (control) or presence of inhibitors $(n=4$ cells for TA, 10 for $9-A C, 7$ for FFA, and 8 for control). Symbols represent mean \pm SEM. (D) Top (patch \#1, representative of 7 out of 9 patches): single-channel currents elicited by $10 \mu \mathrm{M}$ BzATP from an outside-out patch of 16F-null cell transiently transfected with $\mathrm{rP} 2 \mathrm{X} 7$ exhibiting the $\mathrm{O} 1$ state (dashed grey line); c, closed state. Corresponding all points histogram of currents is shown right of the trace. Distributions were fit by the sum of two Gaussians. Bottom (patch \#2, representative of 2 out of 9 patches): example of outside-out patches in which $\mathrm{O} 1$ and an additional high conductance (HC) were recorded (dashed grey lines). Currents were elicited by $10 \mu \mathrm{M}$ BzATP. Corresponding all points histogram of currents is shown right of the trace. Distributions were fit by the sum of three Gaussians. Data were filtered at a final $f_{c}$ of $100 \mathrm{~Hz}$. (E) Summary of corresponding unitary conductances in 16F-null cells. Linking lines indicate data points that originate from the same patches $(n=9$ patches $)$. Bars represent mean \pm SEM. (F) Summary of mean P2X7 unitary conductances in HEK293T and $16 \mathrm{~F}-$ null cells. Mean was calculated by averaging the sum of each state conductance determined from each patch $(n=7$ patches for HEK293T cells and 9 for 16-null cells). Bars represent mean \pm SEM. $P$ value is from Mann-Whitney test. (G) Summary of singlechannel inhibition ( $\mathrm{n}=4$ patches for TA, 3 for 9-AC, 3 for FFA, and 3 for AZ10606120). 
1174 Bars represent mean \pm SEM. Inhibitor concentrations were $20 \mu \mathrm{M}$ TA, $1 \mathrm{mM}$ 9-AC, 100 $1175 \mu \mathrm{M}$ FFA and $1 \mu \mathrm{M}$ AZ10606120. Single-channel and whole-cell data were recorded at 1176120 and $-60 \mathrm{mV}$, respectively. 
1178

1179

1180

1181

1182

1183

1184

1185

1186

1187

1188

1189

1190

1191

1192

1193

1194

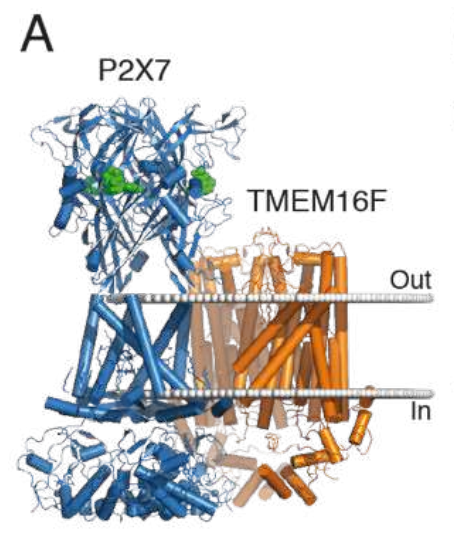

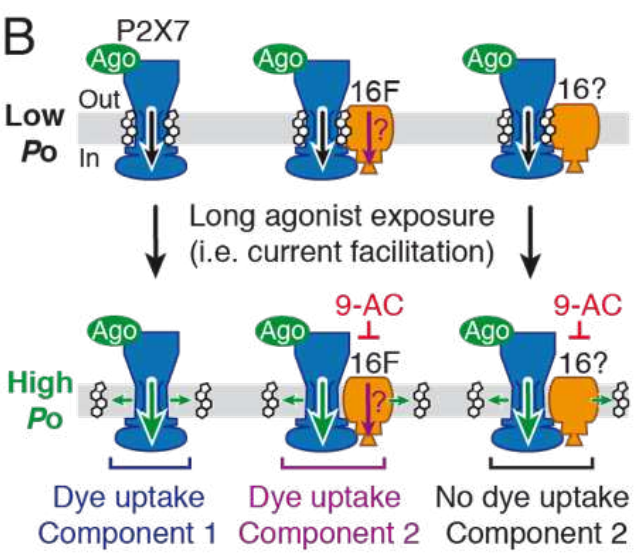

$\begin{array}{ccc}\text { Dye uptake } & \text { Dye uptake } & \text { No dye uptake } \\ \text { Component } 1 & \text { Component } 2 & \text { Component } 2\end{array}$

Figure 8. Proposed mechanisms underlying P2X7 current facilitation and macropore formation. (A) Molecular docking model of ATP-bound rP2X7 (blue, PDB ID: 6u9w) ${ }^{25}$ and $\mathrm{Ca}^{2+}$-bound mTMEM16F (orange, PDB ID: 6qpc) ${ }^{39}$ produced by a Monte-Carlo experiment. Model is shown in cartoon representation and viewed parallel to the membrane plane (delimited by white spheres). ATP is shown as green spheres. (B) Schematic mechanisms of current facilitation and macropore formation. In response to brief application of agonist (green spheres), cholesterol (hexagonal rings) maintains complexembedded P2X7, and possibly "free" P2X7, in a low channel activity state (black arrows). Long or repeated agonist application transitions $\mathrm{P} 2 \mathrm{X} 7$ channels from a low to high $\mathrm{Po}$ (enlarged green arrows), presumably through cholesterol dissociation (small lateral green arrows). Meanwhile, agonist binding to $\mathrm{P} 2 \mathrm{X} 7$ activates at least two permeation components for large molecules, one that occurs directly through the $\mathrm{P} 2 \mathrm{X} 7$ pore (component 1 ), and the other through functional coupling to TMEM16F (16F) (component 2). Note that the precise dye uptake pathway of component 2 (purple arrows) is unknown. Functional coupling with another TMEM16 subtype of unknown composition (16?) is also suggested. Membranes are represented as grey shadings. See text for details. 


\section{Figures}

A

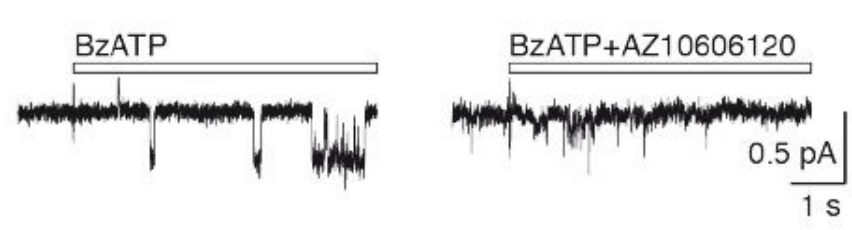

B

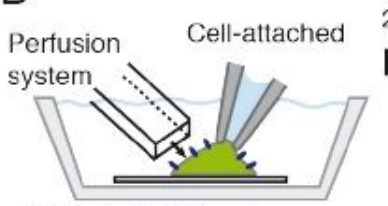

i) Control: NES only

ii) Facilitated: NES+BzATP (30 s)

iii) MCD: NES following MCD incubation
1. Membrane piercing

2. Pipette pulling

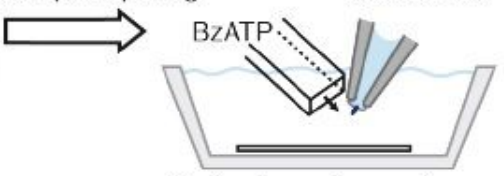

Single-channel recordings
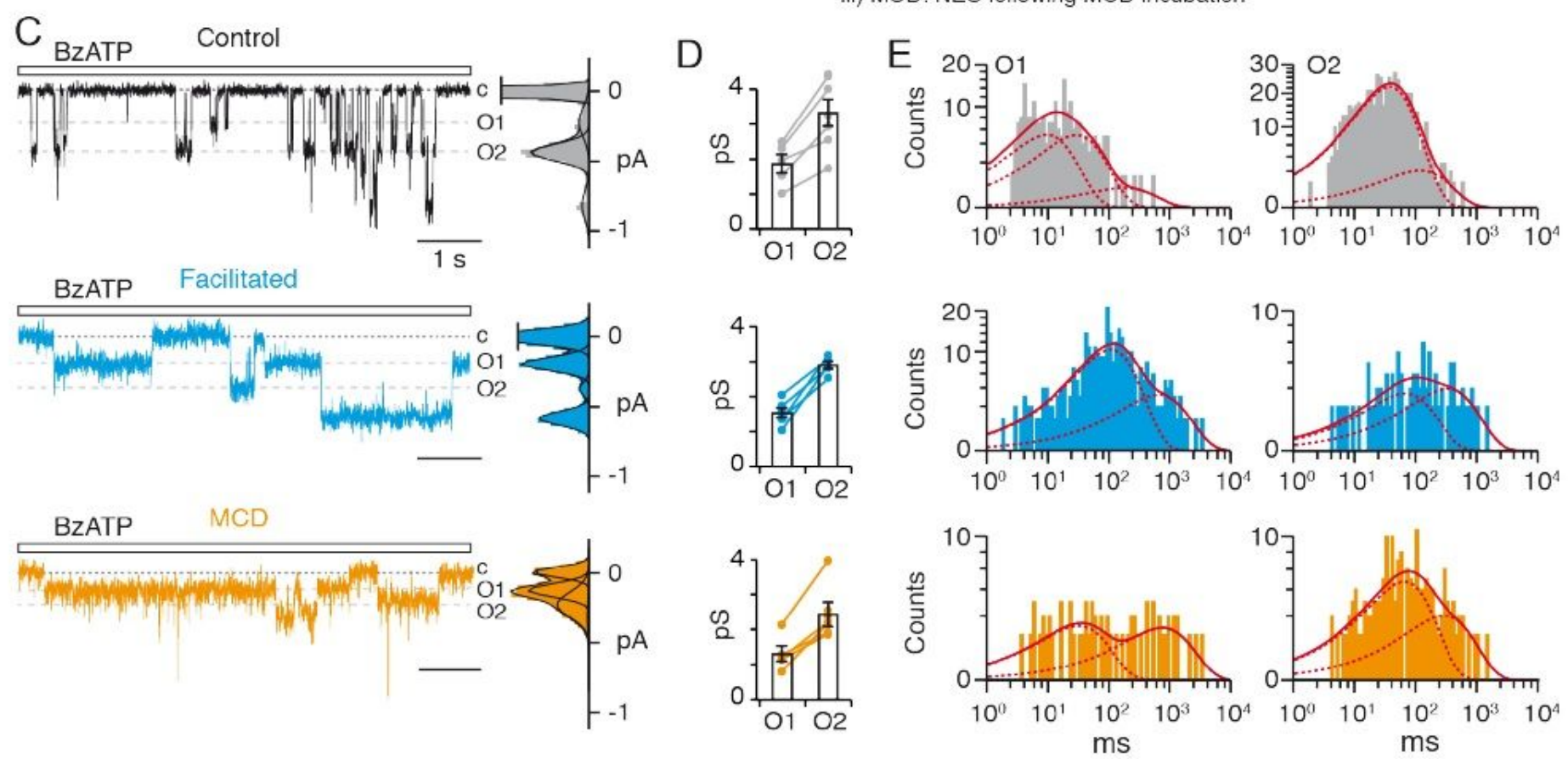

Figure 1

Current facilitation of P2X7 results in an increase of single-channel open probability. (A) Single-channel currents elicited by $10 \mu \mathrm{M}$ BzATP (duration of application indicated by the white bar) from an outside-out patch of HEK293T cell transiently transfected with rP2X7 (left) are inhibited by $1 \mu \mathrm{M} \mathrm{AZ10606120}$ when co applied with $10 \mu \mathrm{M}$ BzATP (right). Traces are from the same patch and channel openings are downward deflections. (B) Cartoon depicting the protocol employed for establishing excised patches from cells expressing rP2X7 (blue ovals) in: i) control conditions, ii) facilitated conditions (10 $\mu \mathrm{M} \mathrm{BzATP}$ for $30 \mathrm{~s}$ ), and iii) MCD-treated conditions. NES: Normal extracellular solution. Figure not to scale. (C) Single-channel currents elicited by $10 \mu \mathrm{M}$ BzATP from outside-out patches of HEK293T cells transiently transfected with rP2X7. Cells were either not treated (black), or pre-treated with a 30 s perfusion of 10 $\mu \mathrm{M}$ BzATP (blue), or incubated with MCD (orange) prior to membrane excising. Dashed grey lines indicate unitary currents corresponding to conductance states 01 and 02. Dotted lines indicate basal current corresponding to closed channels, labeled c. Corresponding all points histograms of currents are shown right of the traces. Distributions were fit by a sum of Gaussians. (D) Summary of corresponding unitary conductances. Linking lines indicate data points that originate from the same patches $(n=7$ patches for control, 6 for facilitated and 7 for MCD-treated conditions). Bars represent mean $\pm S E M$. (E) 
Corresponding open dwell-time histograms fitted by the sum (solid red line) of several exponential functions (dashed red lines). For control patches (grey histograms), t1024 $1=9.6 \mathrm{~ms}$, relative area, a1 $=$ $0.49 ; \mathrm{t} 2=29 \mathrm{~ms}, \mathrm{a} 2=0.48 ; \mathrm{t} 3=180 \mathrm{~ms}, \mathrm{a} 3=0.03$; mean open time, $\mathrm{t} 102501=24 \mathrm{~ms}$ for 01 , and $\mathrm{t} 1=38$ $\mathrm{ms}, \mathrm{a} 1=0.92 ; \mathrm{t} 2=119 \mathrm{~ms}, \mathrm{a} 2=0.08 ; \mathrm{t} 102602=44 \mathrm{~ms}$ for 02. For facilitated (blue histograms), $\mathrm{t} 1=108$ $\mathrm{ms}, \mathrm{a} 1=0.77 ; \mathrm{t} 2=654 \mathrm{~ms}, \mathrm{a} 2=0.23 ; \mathrm{t} 01=234 \mathrm{~ms}$ for 01 and t $1=68 \mathrm{~ms}, \mathrm{a} 1=0.46 ; \mathrm{t} 2=332 \mathrm{~ms}, \mathrm{a} 2=$ $0.54 ; \mathrm{t} 1028 \mathrm{O} 2=211 \mathrm{~ms}$ for 02 . For MCD-treated patches (orange histograms), $\mathrm{t} 1=32 \mathrm{~ms}, \mathrm{a} 1=0.52 ; \mathrm{t} 2=$ $752 \mathrm{~ms}, \mathrm{a} 2=0.48$; $\mathrm{t} 11=378 \mathrm{~ms}$, for 01 and t $1=66 \mathrm{~ms}, \mathrm{a} 1=0.70 ; \mathrm{t} 2=294 \mathrm{~ms}, \mathrm{a} 2=0.30 ; \mathrm{t} 103002=$ $134 \mathrm{~ms}$ for 02. All data were recorded at $-120 \mathrm{mV}$ and filtered at a final fc of $100 \mathrm{~Hz}$.
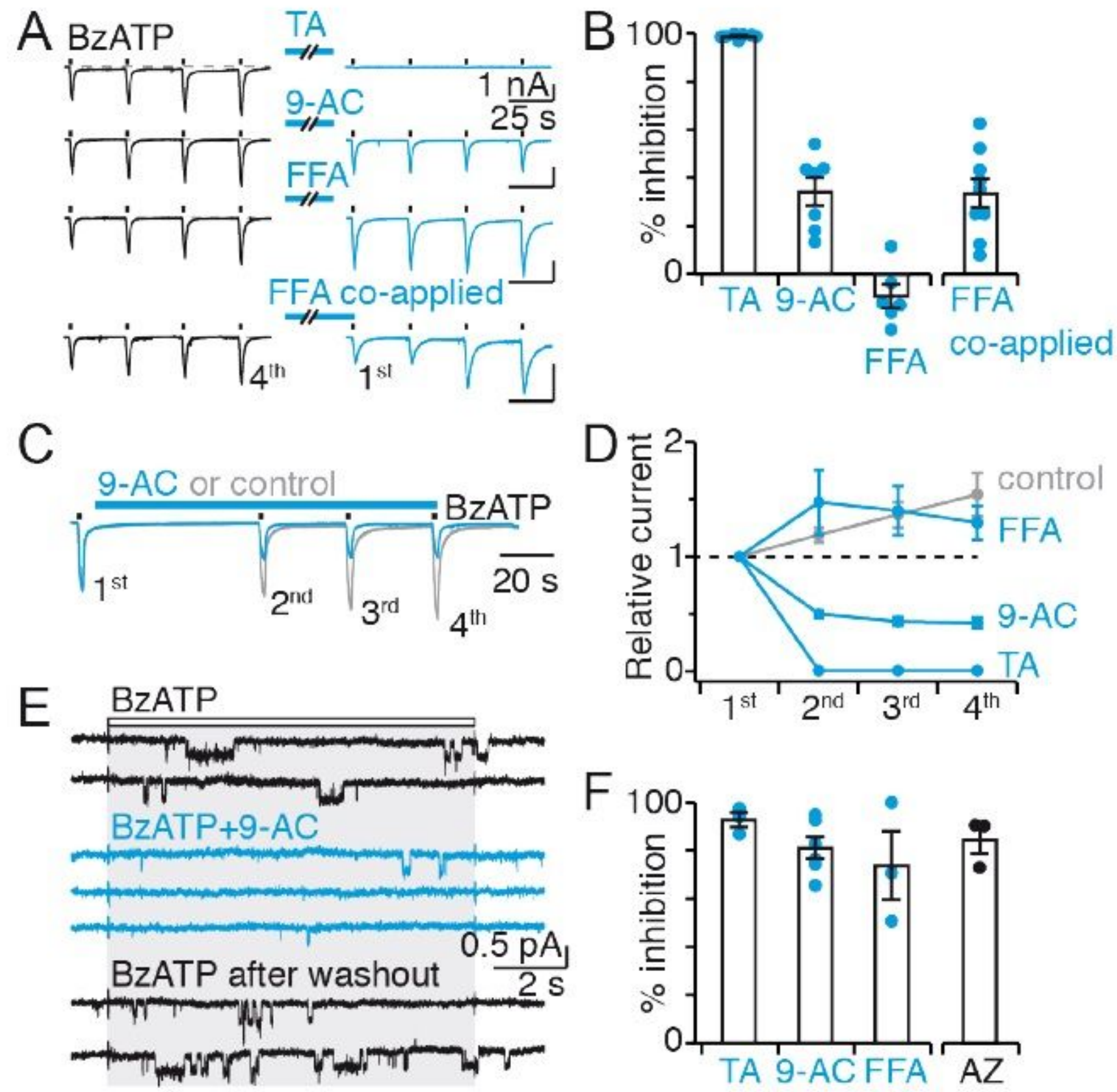

Figure 2

CaCC inhibitors inhibit P2X7 currents in HEK293T cells. (A) Whole-cell currents evoked by repeated $2 \mathrm{~s}$ applications of $10 \mu \mathrm{M}$ BzATP (black bars) before and after $60 \mathrm{~s}$ perfusion of TMEM16 inhibitors (blue 
bars) in HEK293T cells transiently expressing rP2X7. In the case of FFA co-applied, perfusion was followed by a co-application with $10 \mu \mathrm{M}$ BzATP. (B) Summary of whole-cell inhibition ( $\mathrm{n}=7$ cells for TA, 7 for 9 -AC, 6 for FFA, and 9 for FFA co-applied). Inhibition is calculated by comparing current amplitude of the fourth application before and first application after perfusion. Bars represent mean \pm SEM. (C) Wholecell currents evoked by repeated $2 \mathrm{~s}$ applications of $10 \mu \mathrm{M}$ BzATP in the presence (blue) or absence of 9AC (grey) in HEK293T cells transiently expressing rP2X7. Currents are normalized to the first application. (D) Summary of currents relative to the first application in the absence (control) or presence of inhibitors $(n=4$ cells for TA, 10 for $9-A C, 8$ for FFA, and 8 for control). Symbols represent mean \pm SEM. (E)

Single channel rP2X7 currents elicited by $10 \mu \mathrm{M}$ BzATP from an outside-out patch of HEK293T cell (black traces) are inhibited by $1 \mathrm{mM}$ 9-AC when co-applied with $10 \mu \mathrm{M}$ BzATP (blue traces). Shown sweeps were sequentially recorded from the same patch. Note that inhibition is reversible after washout. Duration of application is indicated by the white bar and grey shading. Data were filtered at a final fc of $100 \mathrm{~Hz}$. (F) Summary of single-channel inhibition ( $n=3$ patches for TA, 6 for 9-AC, 3 for FFA, and 3 for AZ10606120). Bars represent mean $\pm S E M$. See Materials and Methods for calculation. Inhibitor concentrations were 20 $\mu \mathrm{M}$ TA, 1 mM 9-AC, $100 \mu \mathrm{M}$ FFA and $1 \mu \mathrm{M}$ AZ10606120. Single channel and whole-cell data were recorded at -120 and $-60 \mathrm{mV}$, respectively.
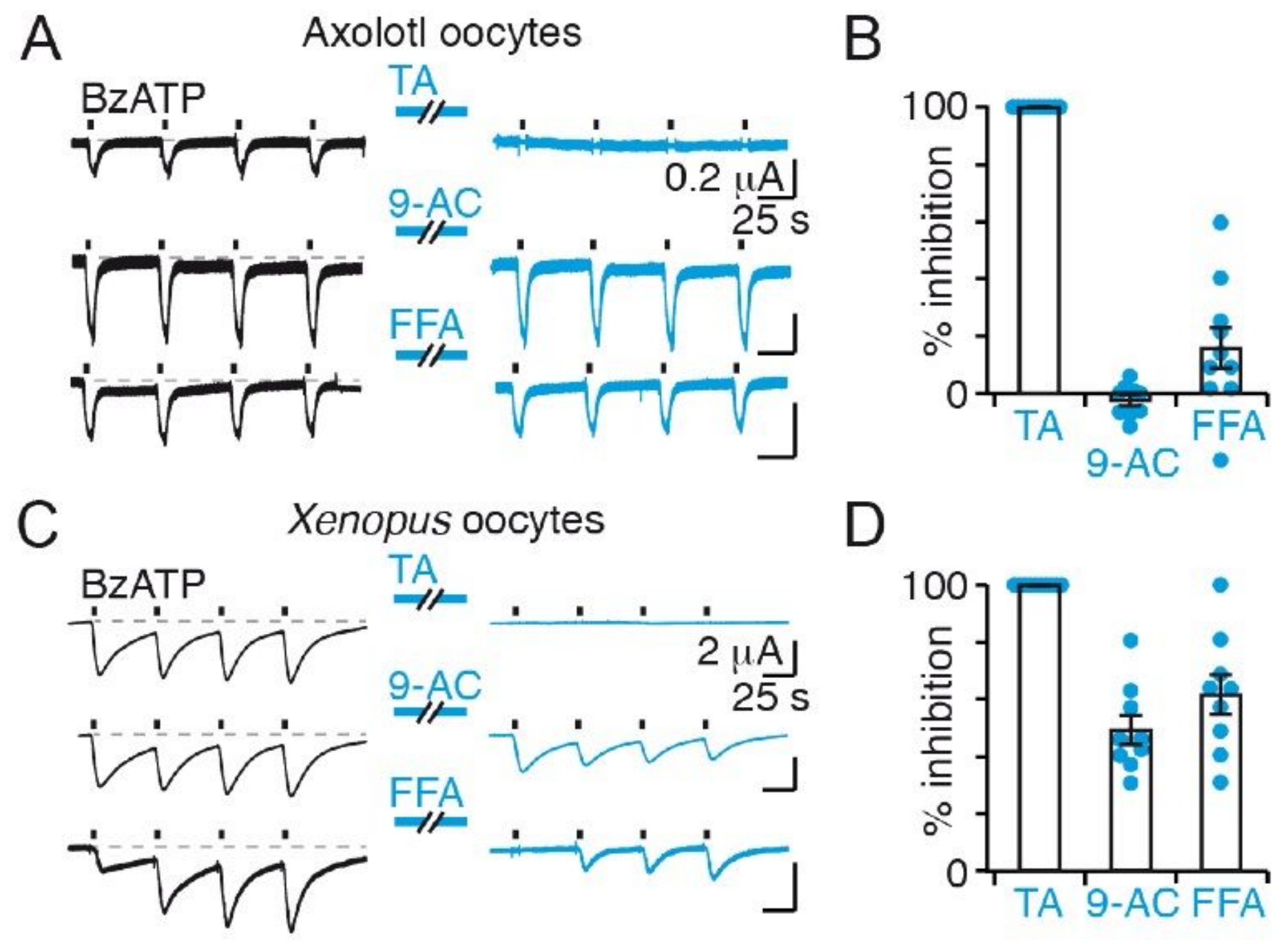

Figure 3

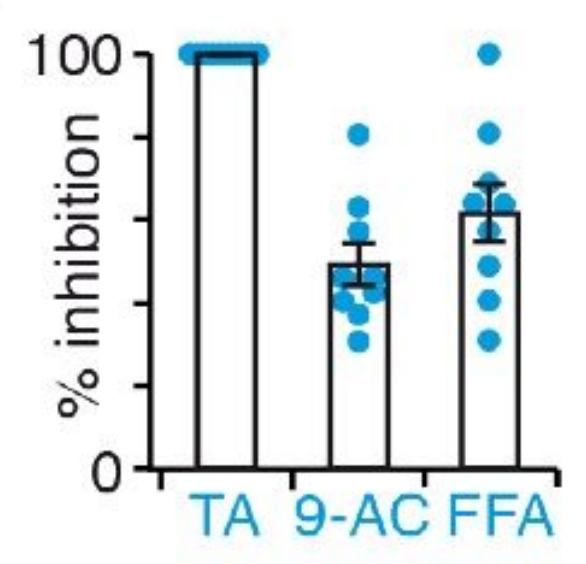


9-AC and FFA do not inhibit P2X7 currents in Axolotl. (A) Whole-cell BzATP-evoked currents recorded by TEVC of Axolotl oocytes expressing rP2X7 before and after application of TMEM16 inhibitors using the same protocol as in Fig. 2A. (B) Summary of inhibition in Axolotl oocytes calculated as in Fig. $2 B(n=10$ oocytes for TA, 9 for 9-AC, and 10 for FFA). Bars represent mean \pm SEM. (C) Whole-cell BzATPevoked currents recorded by TEVC of Xenopus oocytes expressing rP2X7 using the same protocol as in Fig. 2A. (D) Summary of inhibition in Xenopus oocytes calculated as in Fig. $2 B(n=10$ oocytes for TA, 9 for $9-A C$, and 9 for FFA). Bars represent mean \pm SEM. CaCC inhibitor concentrations were $20 \mu M$ TA, 1 $\mathrm{mM} 9-\mathrm{AC}$ and $100 \mu \mathrm{M}$ FFA. Data were recorded at $-60 \mathrm{mV}$.

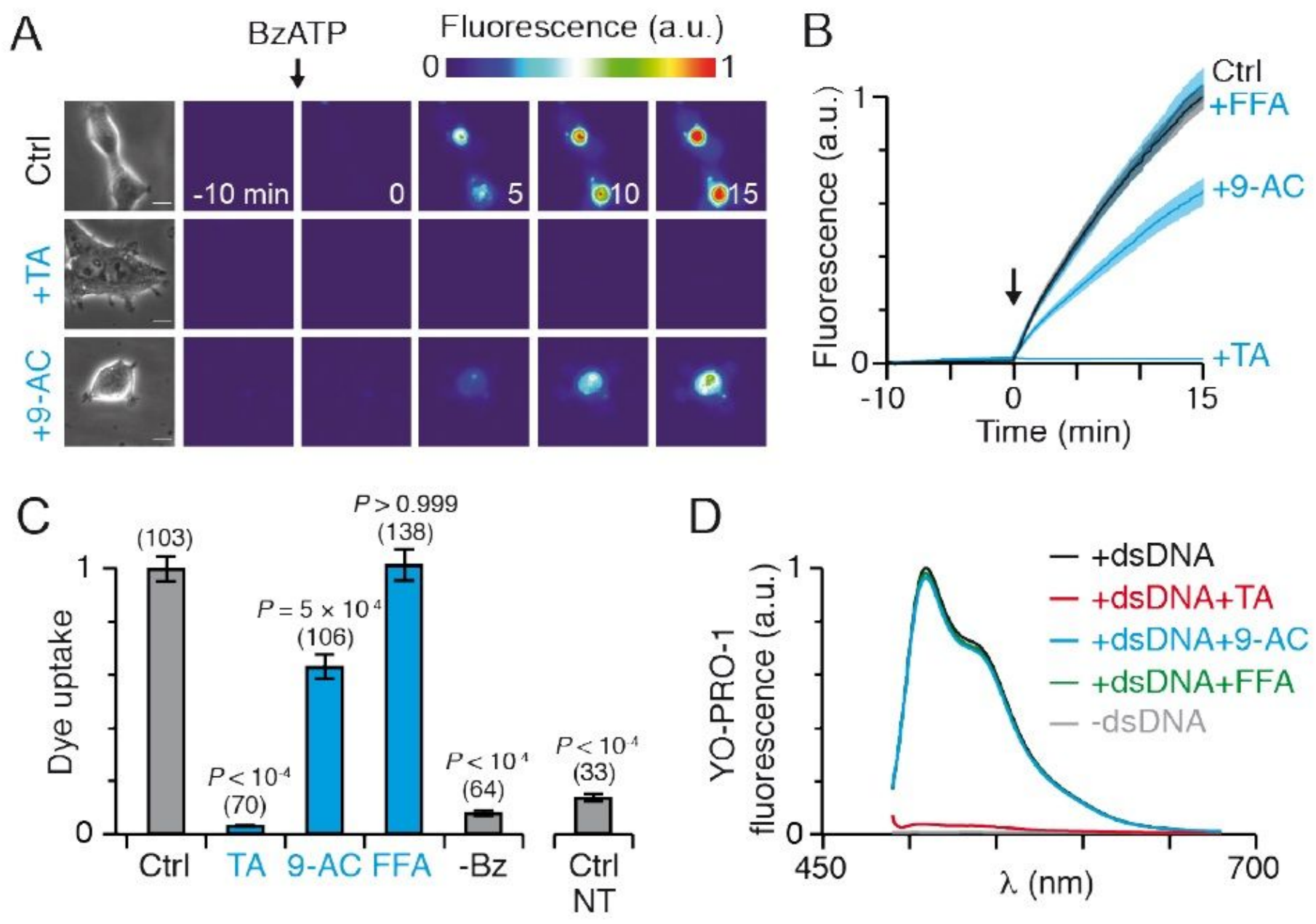

Figure 4

Effects of CaCC inhibitors on dye uptake. (A) Time series fluorescence images of YO-PRO-1 dye uptake in HEK293T cells transiently expressing $\mathrm{rP2X7}$, in the absence or presence of inhibitors, as indicated. Fluorescence (in arbitrary units) was acquired at indicated times before and after $10 \mu \mathrm{M}$ BzATP (arrow) application, which begins at 0 min. Inhibitors were co-applied at the same time as BzATP. On the left are shown the corresponding microphotographs under transmitted light (scale bar, $10 \mu \mathrm{m}$ ).

(B) Corresponding YO-PRO-1 dye uptake over time upon BzATP activation (arrow) in the absence or presence of inhibitors, as indicated. Solid lines represent mean \pm SEM (shaded areas). (C) Summary of BzATP-induced YO-PRO-1 dye uptake normalized to rP2X7-transfected cells in the absence (Ctrl, grey 
histogram) or presence of inhibitors (blue histograms). Numbers of cells are indicated above bars. Dye uptake in the absence of BzATP is labelled -Bz. BzATP-induced dye uptake in non-transfected cells (NT) was normalized to another set of rP2X7-transfected control $(n=78$ cells). Bars represent mean \pm SEM (from 3 to 12 transfections). P values are from one-way Kruskal-Wallis ANOVA followed by Dunn's comparison to respective controls. (D) Fluorescence spectra of $10 \mu \mathrm{M}$ YO-PRO-1 bound to double strand DNA (dsDNA) recorded before (black trace, averaged from respective controls) and immediately after application of TA (red), 9-AC (blue) or FFA (green). Fluorescence spectra of $10 \mu \mathrm{M}$ unbound YO-PRO-1 (in the absence of dsDNA) is indicated in grey. CaCC inhibitor concentrations were $20 \mu \mathrm{M} \mathrm{TA}, 1 \mathrm{mM}$ 9-AC and $100 \mu \mathrm{M}$ FFA.
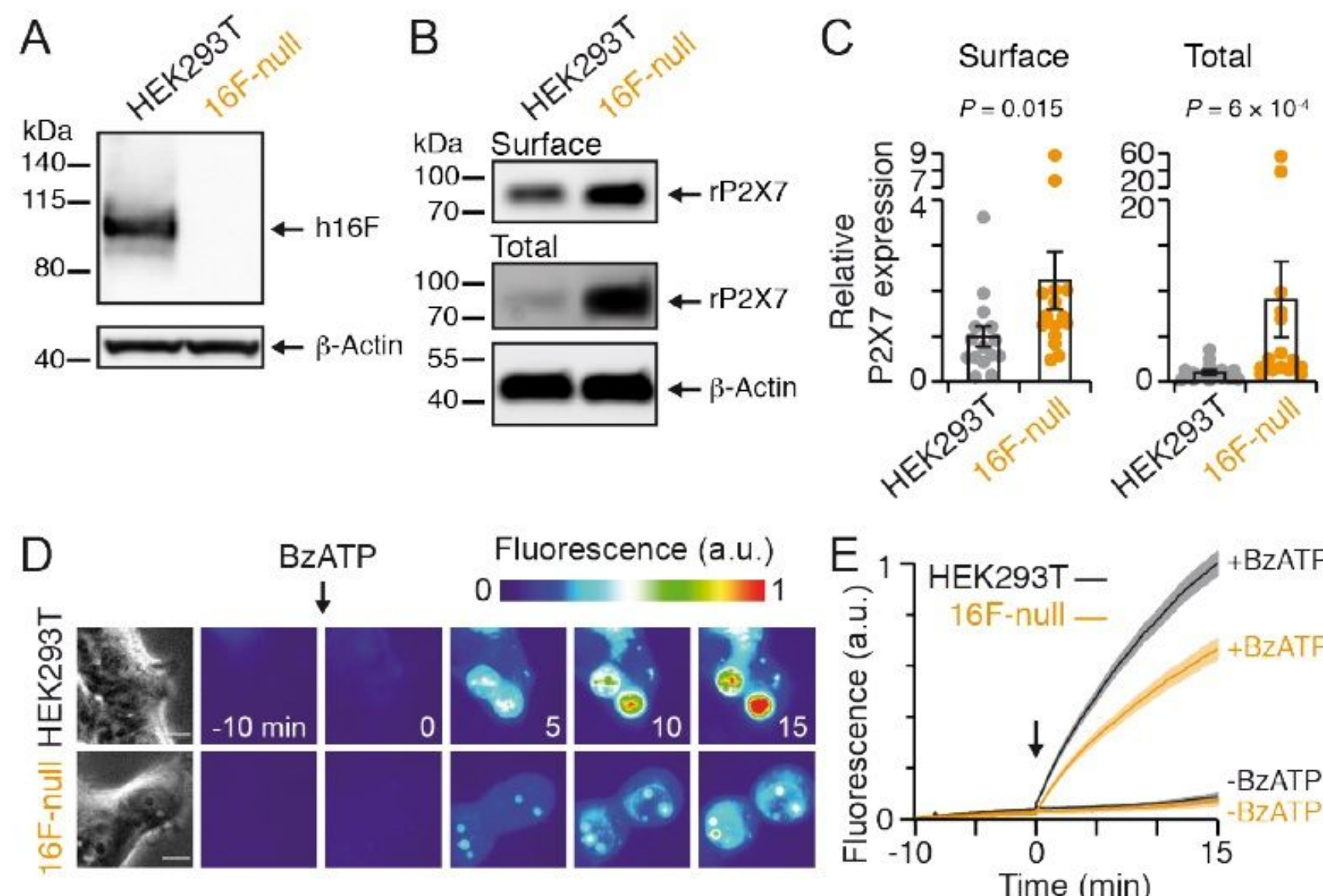

$\mathrm{F}$
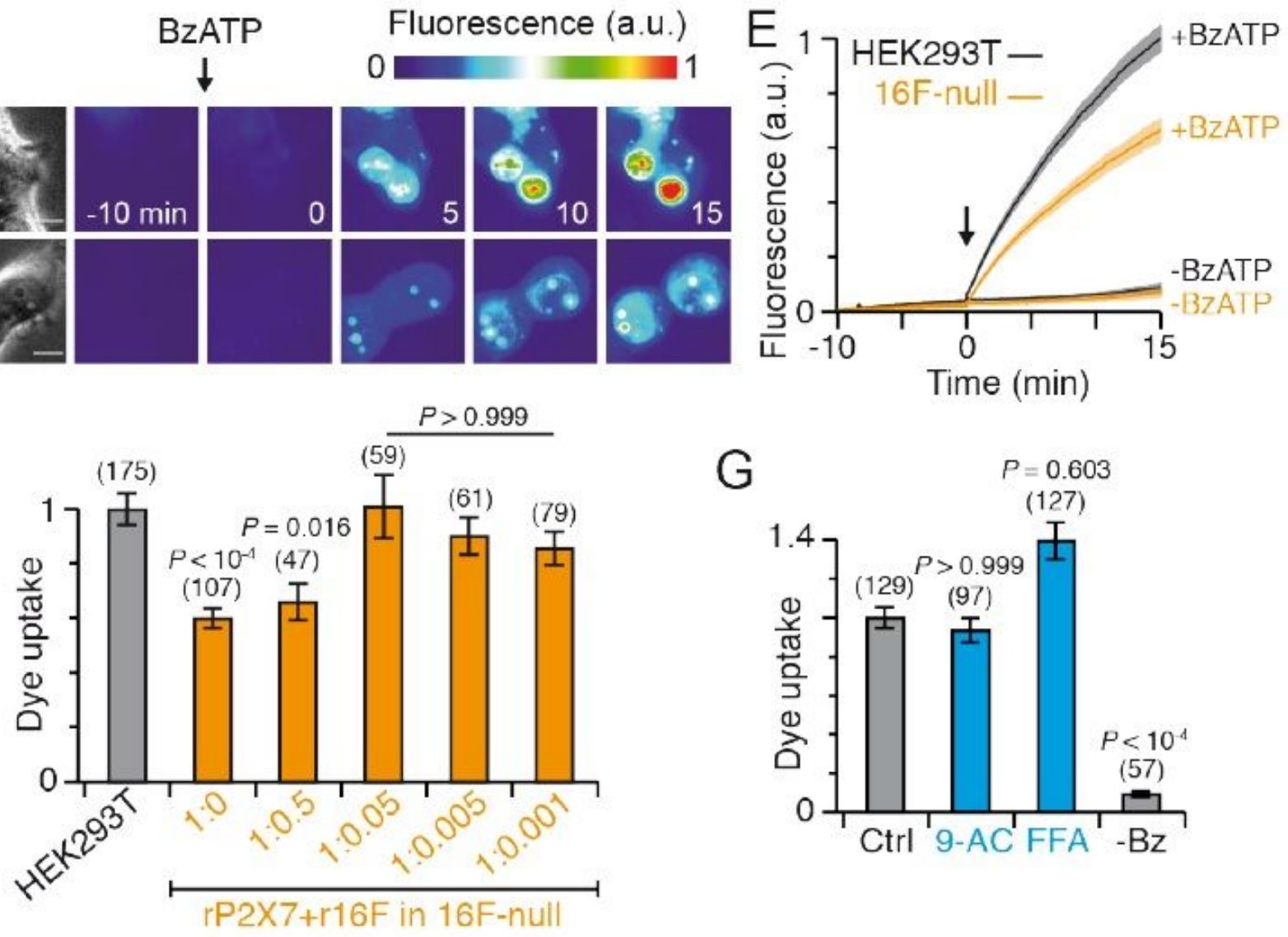

G

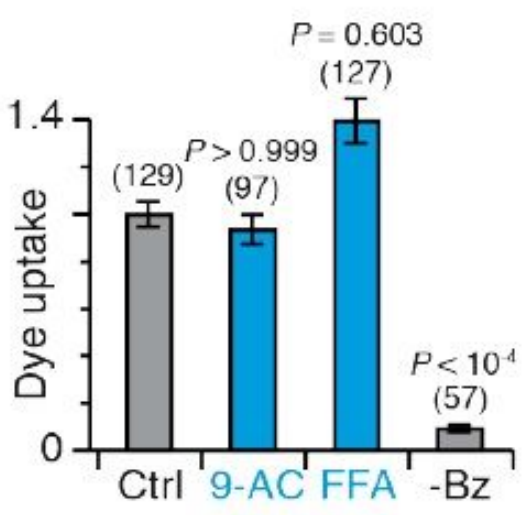




\section{Figure 5}

TMEM16F contributes to dye uptake. (A) Western blot analysis of total cell lysate separated by SDS-PAGE indicates the successful knock-out of endogenous hTMEM16F (h16F) by CRISPR/Cas9 in 16F-null cells. Western blotting was performed with anti-hTMEM16F antibody. b-Actin was used as a loading control. (B) Total and surface expression of myc tagged rP2X7 subunits transiently expressed in HEK293T or in 16F-null cells and resolved by SDS-PAGE. Proteins at the cell surface were biotinylated and isolated with neutravidin-bound agarose resin. Western blotting was performed with anti-c-myc antibody. b-Actin was used as a loading control. Apparent molecular weight markers are indicated. Uncropped blots are shown in Fig. S6A and B. (C) Summary of relative P2X7 expression normalized to b-actin. Bars represent mean \pm SEM (from 15 transfections). P values are from Mann-Whitney test. (D) Time series fluorescence images of YO-PRO-1 dye uptake upon $10 \mu \mathrm{M}$ BzATP activation (arrow) in HEK293T cells and 16F-null cells transiently expressing rP2X7. See legend of Fig. 4A for details. Scale bar, $10 \mu \mathrm{m}$. (E) Corresponding YO-PRO-1 uptake over time in HEK293T cells (black) and 16F-null cells (orange), in the absence (-BzATP) or presence of $10 \mu \mathrm{M}$ BzATP (+BzATP) applied as indicated (arrow). Solid lines represent mean \pm SEM (shaded areas). (F) Summary of BzATP-induced YO-PRO-1 uptake in 16F-null cells transiently expressing rP2X7 $(2 \mu \mathrm{g})$ in the absence (ratio 1:0) or presence of rTMEM16F, 1108 transfected with varying quantities of cDNA (from 1 to $0.002 \mu \mathrm{g}$ ) at the indicated ratios (defined as rP2X7 to TMEM16F). Shown is the specific dye uptake, found by subtracting residual uptake recorded in the absence of BzATP. Data are normalized to rP2X7-expressing HEK293T cells. Bars represent mean \pm SEM, and numbers of cells are indicated above bars (from 4 to 5 transfections). P values are from one-way Kruskal-Wallis ANOVA followed by Dunn's comparison to HEK293T. (G) Summary of YO-PRO-1 uptake in 16F-null cells in the absence (Ctrl) or presence of TMEM16 inhibitors. Dye uptake in the absence of BzATP is labeled -Bz. Bars represent mean $\pm S E M$, and numbers of cells are indicated above bars (from 5 transfections). $P$ values are from one-way Kruskal-Wallis ANOVA followed by Dunn's comparison to control (Ctrl). CaCC inhibitor concentrations were $1 \mathrm{mM} 9-\mathrm{AC}$ and $100 \mu \mathrm{M}$ FFA. 


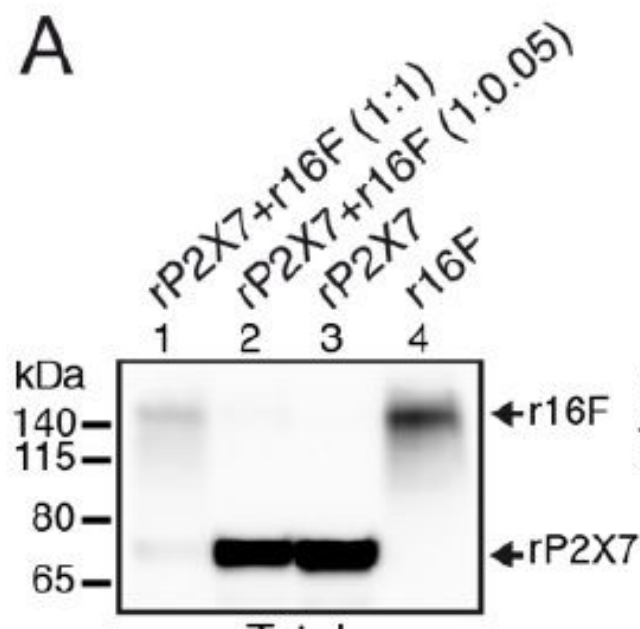

Total

WB: @c-myc
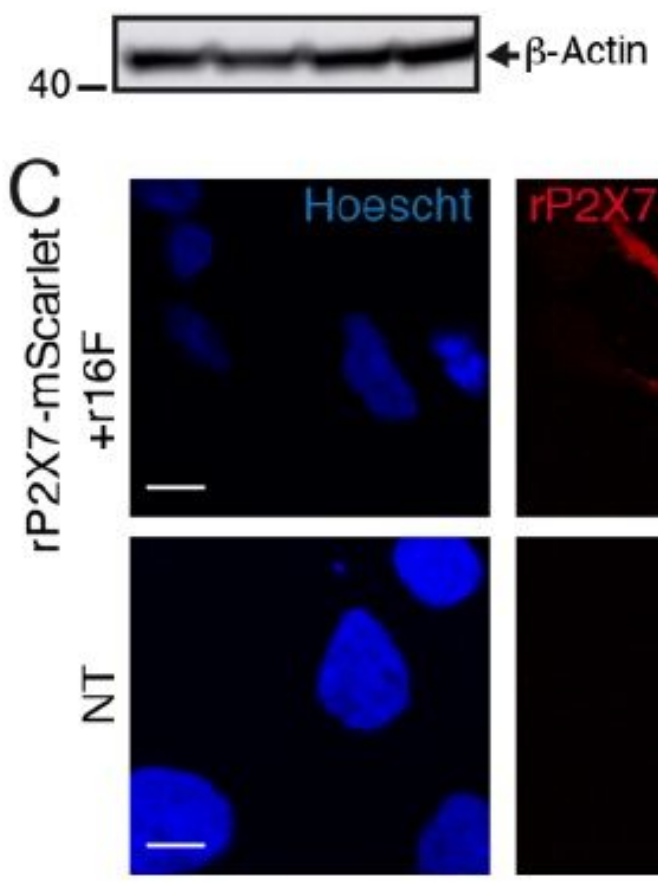
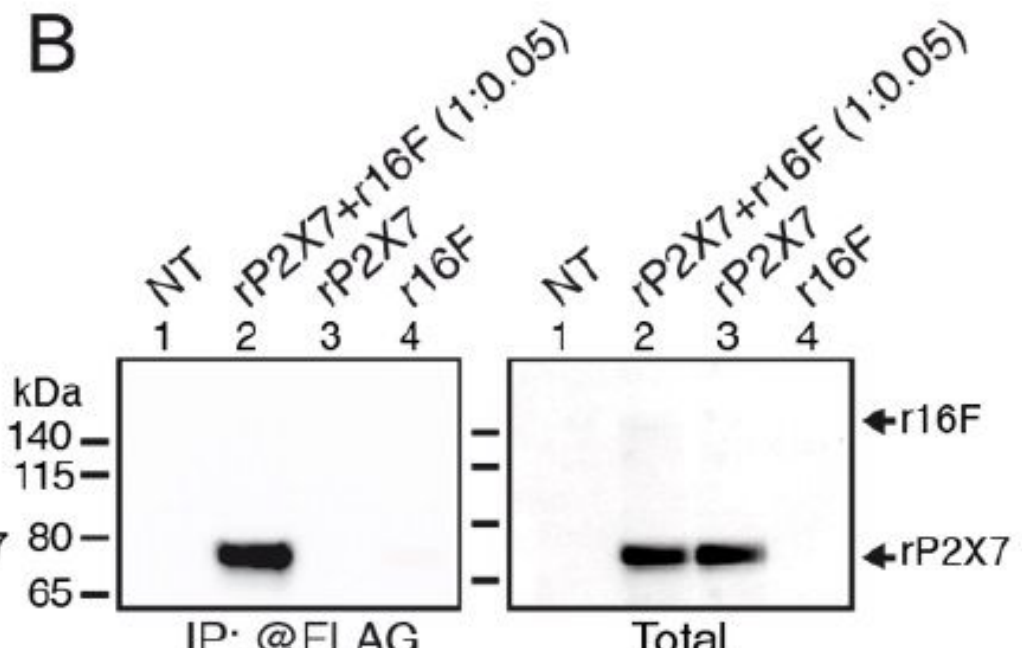

Total

WB: @c-myc

WB: @c-myc
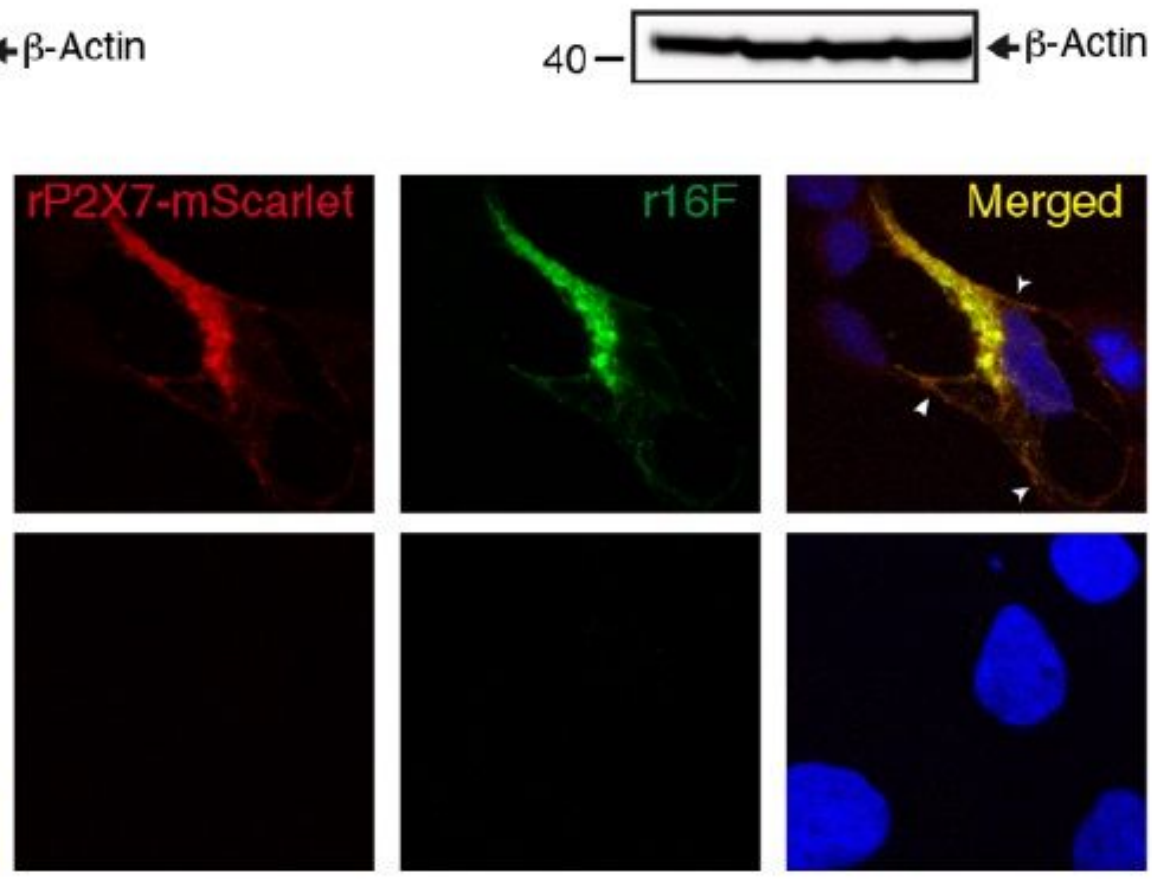

Figure 6

Close physical proximity of P2X7 and TMEM16F. (A) Western blot of total cell lysate separated by SDSPAGE from 16F-null cells transiently transfected with myc tagged rP2X7 (rP2X7) and myc-DDK tagged rTMEM16F (r16F) at a ratio of 1:1 (lane 1: $5 \mu \mathrm{g}$ rP2X7 and $5 \mu \mathrm{g} \mathrm{r16F}$ ) or 1:0.05 (lane 2: $5 \mu \mathrm{g} \mathrm{rP2X7}$ and $0.25 \mu \mathrm{g} \mathrm{r} 16 \mathrm{~F}$ ) and probed with anti-c-myc antibody. Note reduced rP2X7 subunit expression in lane 1 compared to that in lane 2. Control expressions of rP2X7 $(10 \mu \mathrm{g})$ and $\mathrm{r} 16 \mathrm{~F}(10 \mu \mathrm{g})$ are shown in lane 3 and 4 , respectively. b-Actin was used as a loading control. Apparent molecular weight markers are indicated. (B) Left: Co-immunoprecipitation reveals physical association of myc tagged rP2X7 and mycDDK tagged r16F subunits transiently co-expressed at a ratio of 1:0.05 (5 $\mu \mathrm{g} \mathrm{rP2X7}$ and $0.25 \mu \mathrm{g} \mathrm{r16F})$ in $16 \mathrm{~F}$-null cells and resolved by SDS-PAGE. Co-immunoprecipitation was performed in cell lysate with anti- 
FLAG antibody, which recognizes DDK tag of myc-DDK tagged r16F, and Western blotting was performed with anti-c-myc antibody. Right: Western blot of total cell lysate of corresponding samples probed with anti-c-myc antibody. Control expressions of rP2X7 (5 $\mu \mathrm{g})$ and r16F $(0.25 \mu \mathrm{g})$ are shown in lanes 3 and 4 , respectively. Note that the band corresponding to myc-DDK tagged $\mathrm{r} 16 \mathrm{~F}$ is barely visualized because of the 20-fold lower expression of myc-DDK tagged r16F compared to that of rP2X7 (see, however, Fig. S6D for the actual presence of $\mathrm{r} 16 \mathrm{~F}$ ). Controls in non-transfected (NT) cells are shown in lanes 1. b-Actin is used as the loading control. Uncropped blots are shown in Fig. S6C and D. (C) Top: Confocal images of 16F-null cells transiently transfected with $2 \mu \mathrm{g} \mathrm{rP2X7-mScarlet} \mathrm{(red)} \mathrm{and} 0.1 \mu \mathrm{g} \mathrm{r16F}$ (green) demonstrate co-localization (yellow) of both proteins. Head arrows indicate putative cell membrane localizations. Cells were stained with a primary anti-h $16 \mathrm{~F}$ antibody coupled to a secondary Alexa 488 conjugated antibody (green). Nuclei 1143 were stained with Hoescht (blue). Bottom: Control in non-transfected (NT) 16F-null cells. Scale bar, $10 \mu \mathrm{m}$.
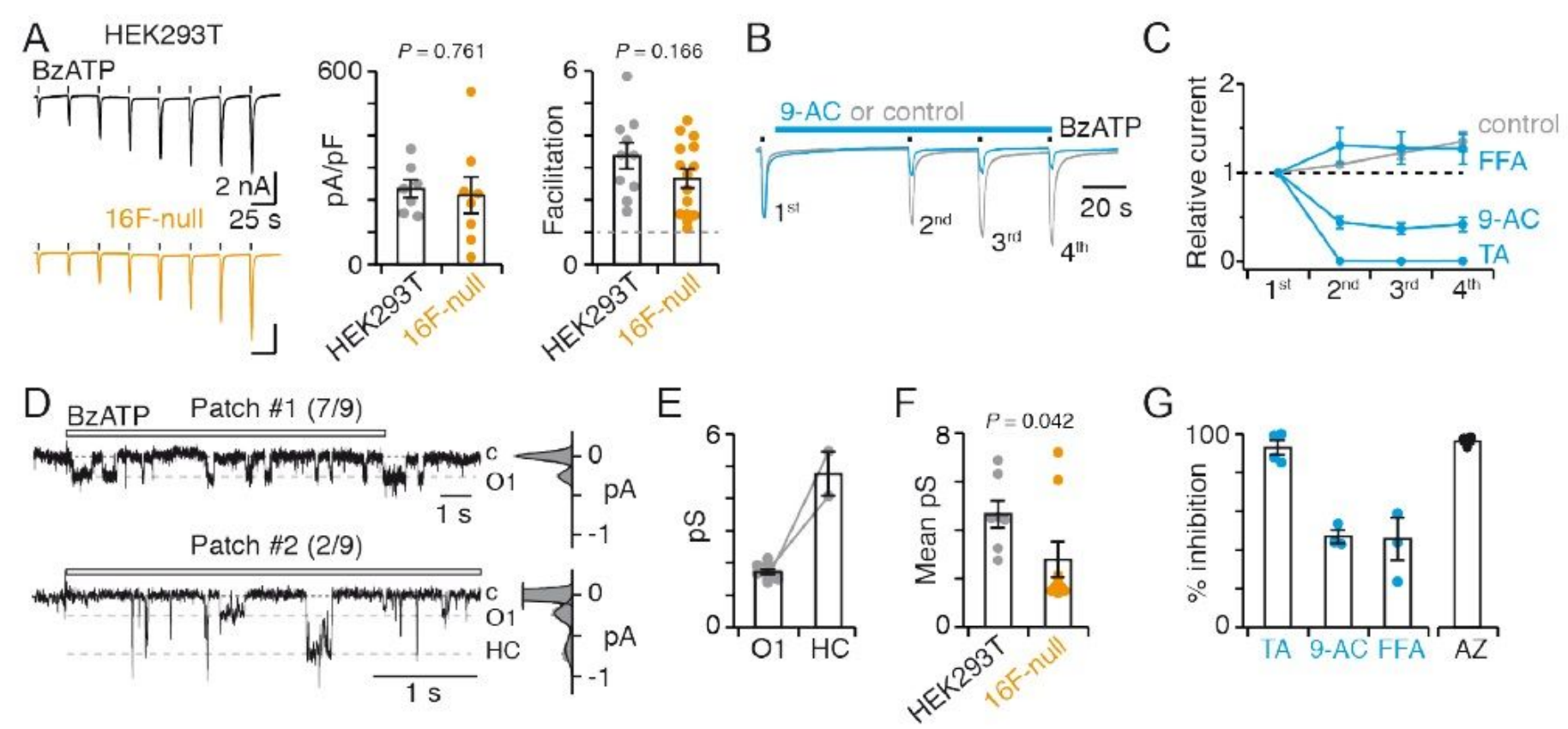

\section{Figure 7}

TMEM16F shapes P2X7 unitary conductance. (A) Left: whole-cell currents evoked by repeated $2 \mathrm{~s}$ applications of $10 \mu \mathrm{M}$ BzATP recorded in HEK293T (black trace) and 16F-null cells (orange trace) transiently transfected with rP2X7. Middle: summary of current density of the 8th application ( $\mathrm{n}=7$ for HEK293T cells and 8 for 16F-null cells). Bars represent mean \pm SEM. Right: summary of current facilitation determined between the 8th and 1st application. See Materials and Methods for calculation ( $\mathrm{n}$ $=10$ cells for HEK293T and 15 for 16F-null). P values are from unpaired Student's t-test. (B) Whole cell currents evoked by repeated $2 \mathrm{~s}$ applications of $10 \mu \mathrm{M}$ BzATP in the presence (blue) or absence of 9-AC (grey) in 16F-null cells transiently expressing rP2X7. Currents are normalized to the first application. (C) Summary currents relative to the first application in the absence (control) or presence of inhibitors $(n=4$ cells for TA, 10 for 9-AC, 7 for FFA, and 8 for control). Symbols represent mean \pm SEM. (D) Top (patch \#1, representative of 7 out of 9 patches): single-channel currents elicited by $10 \mu \mathrm{M}$ BzATP from an outside- 
out patch of 16F-null cell transiently transfected with rP2X7 exhibiting the 01 state (dashed grey line); , closed state. Corresponding all points histogram of currents is shown right of the trace. Distributions were fit by the sum of two Gaussians. Bottom (patch \#2, representative of 2 out of 9 patches): example of outside-out patches in which 01 and an additional high conductance $(\mathrm{HC})$ were recorded (dashed grey lines). Currents were elicited by $10 \mu \mathrm{M}$ BzATP. Corresponding all points histogram of currents is shown right of the trace. Distributions were fit by the sum of three Gaussians. Data were filtered at a final fc of $100 \mathrm{~Hz}$. (E) Summary of corresponding unitary conductances in 16F-null cells. Linking lines indicate data points that originate from the same patches $(n=9$ patches). Bars represent mean \pm SEM. ( $F)$ Summary of mean P2X7 unitary conductances in HEK293T and 16F-null cells. Mean was calculated by averaging the sum of each state conductance determined from each patch $(n=7$ patches for HEK293T cells and 9 for 16 -null cells). Bars represent mean \pm SEM. $P$ value is from Mann-Whitney test. (G) Summary of single channel inhibition ( $n=4$ patches for TA, 3 for 9-AC, 3 for FFA, and 3 for AZ10606120).Bars represent mean \pm SEM. Inhibitor concentrations were $117420 \mu \mathrm{M}$ TA, $1 \mathrm{mM}$ 9-AC, $100 \mu \mathrm{M}$ FFA and $1 \mu \mathrm{M}$ AZ10606120. Single-channel and whole-cell data were recorded at 120 and $-60 \mathrm{mV}$, respectively.
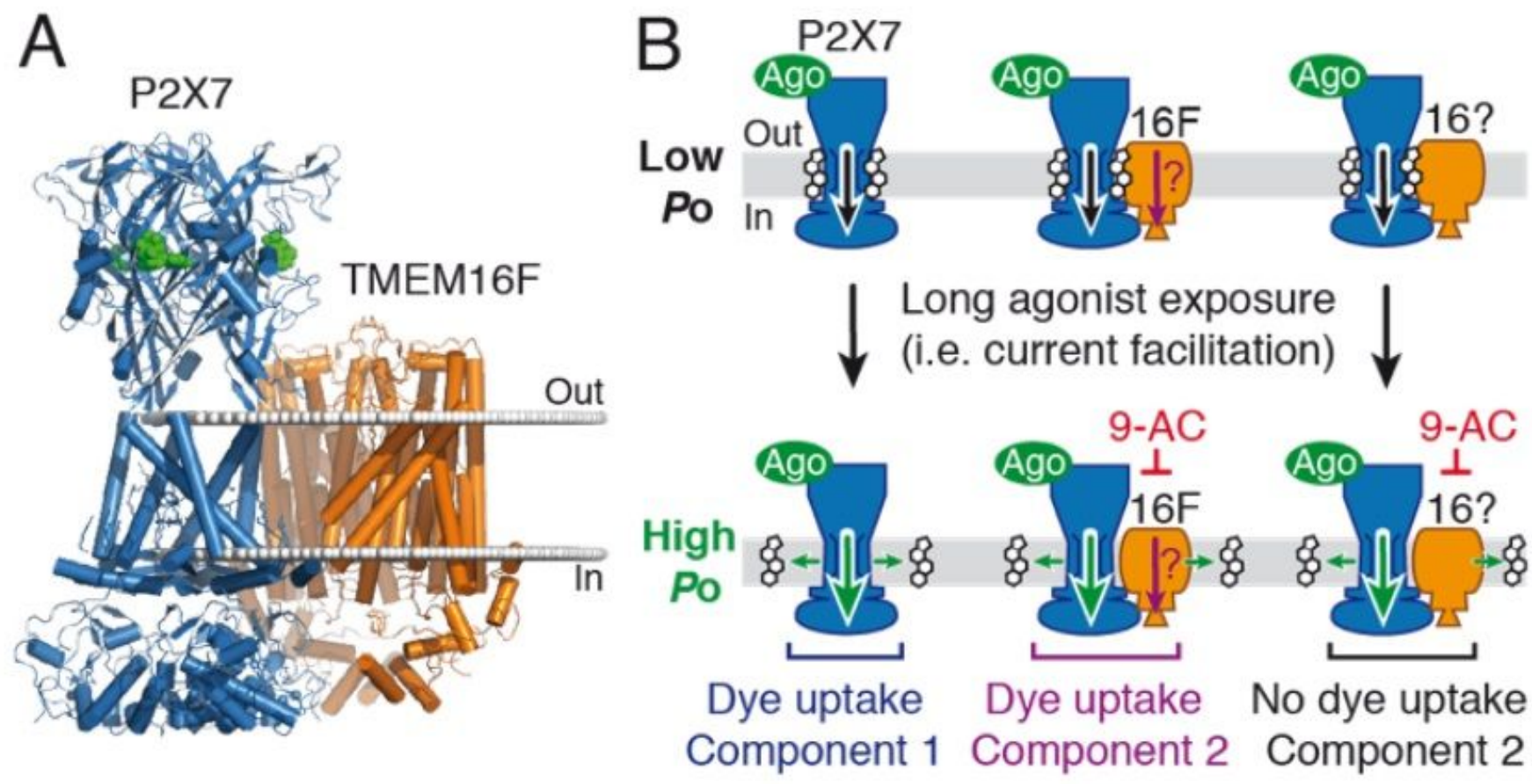

Figure 8

Proposed mechanisms underlying P2X7 current facilitation and macropore formation. (A) Molecular docking model of ATP-bound rP2X7 (blue, PDB ID: 6u9w) 25 and Ca2+-bound mTMEM16F (orange, PDB ID: 6qpc)39 produced by a Monte-Carlo experiment. Model is shown in cartoon representation and viewed parallel to the membrane plane (delimited by white spheres). ATP is shown as green spheres. (B) Schematic mechanisms of current facilitation and macropore formation. In response to brief application of agonist (green spheres), cholesterol (hexagonal rings) maintains complex embedded P2X7, and possibly "free" P2X7, in a low channel activity state (black arrows). Long or repeated agonist application 
transitions P2X7 channels from a low to high Po (enlarged green arrows), presumably through cholesterol dissociation (small lateral green arrows). Meanwhile, agonist binding to P2X7 activates at least two permeation components for large molecules, one that occurs directly through the $\mathrm{P} 2 \mathrm{X} 7$ pore (component 1), and the other through functional coupling to TMEM16F (16F) (component 2). Note that the precise dye uptake pathway of component 2 (purple arrows) is unknown. Functional coupling with another TMEM16 subtype of unknown composition (16?) is also suggested. Membranes are represented as grey shadings. See text for details.

\section{Supplementary Files}

This is a list of supplementary files associated with this preprint. Click to download.

- SIAppendixCOMMBIOL.docx 